\title{
Real-time Integration of Building Energy Data
}

\section{Diogo Anjos}

Thesis to obtain the Master of Science Degree in Information Systems and Computer Engineering

November 20, 2015 


\section{Agenda}

- Introduction

- State of the art

- Motivation

- Problem Statement

- Proposed Solution

- Evaluation

- Conclusion

- Bibliography 


\section{Agenda}

- Introduction

- State of the art

- Motivation

- Problem Statement

- Proposed Solution

- Evaluation

- Conclusion

- Bibliography 


\section{Introduction}

Three ways to improve Energy Efficiency in Buildings

[Pérez-Lombard et al., 2008] 


\section{Introduction}

\section{Three ways to improve Energy Efficiency in Buildings}

[Pérez-Lombard et al., 2008]

\section{Energy \\ Conservation}

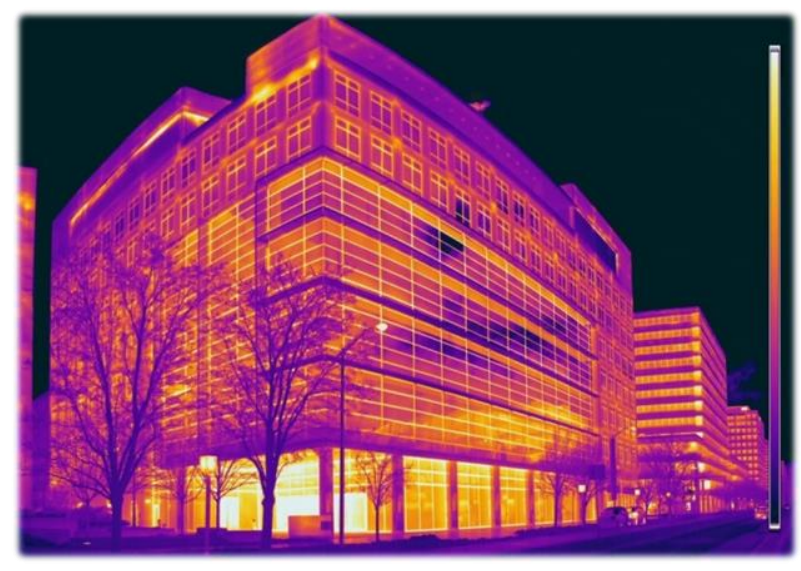

MIT Field Intelligence Laboratory 


\section{Introduction}

\section{Three ways to improve Energy Efficiency in Buildings}

[Pérez-Lombard et al., 2008]

Energy

Conservation

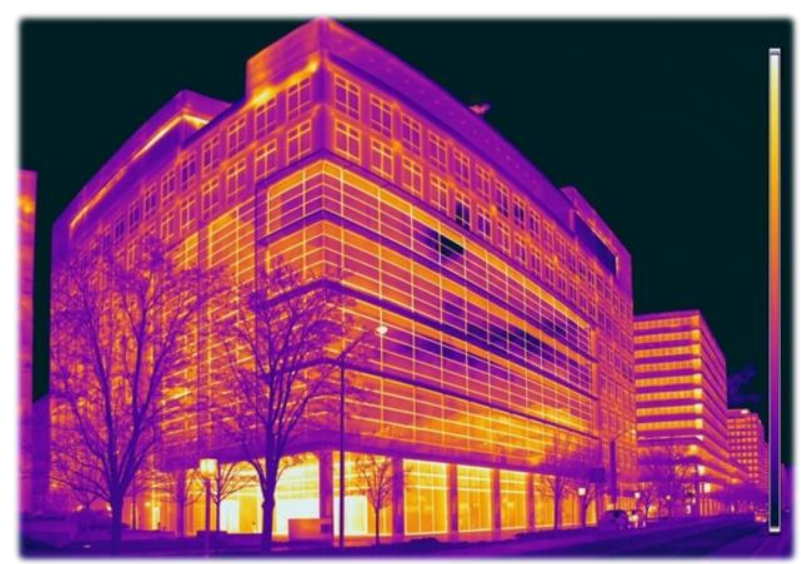

MIT Field Intelligence Laboratory
Equipment

Efficiency

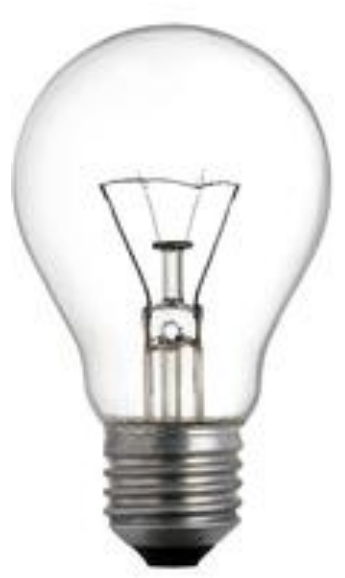

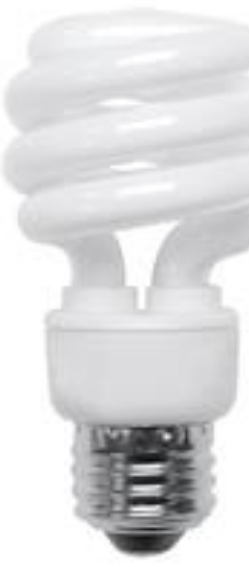




\section{Introduction}

\section{Three ways to improve Energy Efficiency in Buildings}

[Pérez-Lombard et al., 2008]

Energy

Conservation

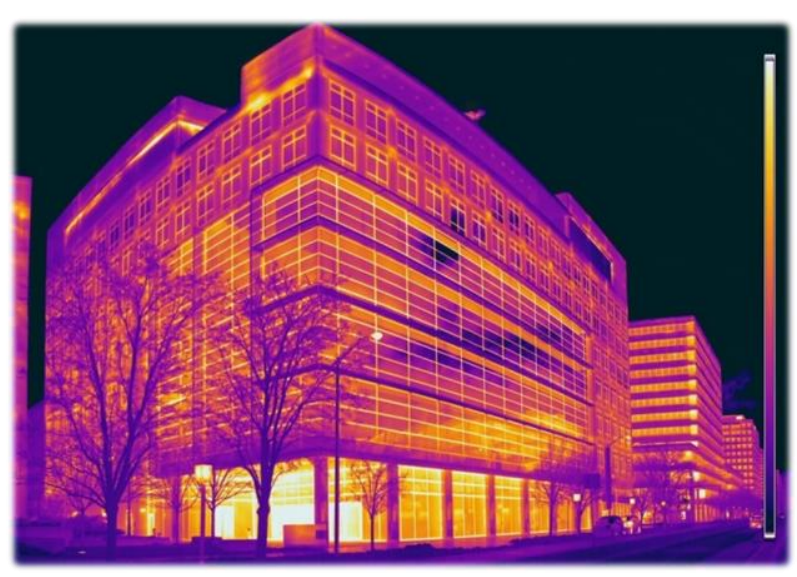

MIT Field Intelligence Laboratory
Equipment

Efficiency

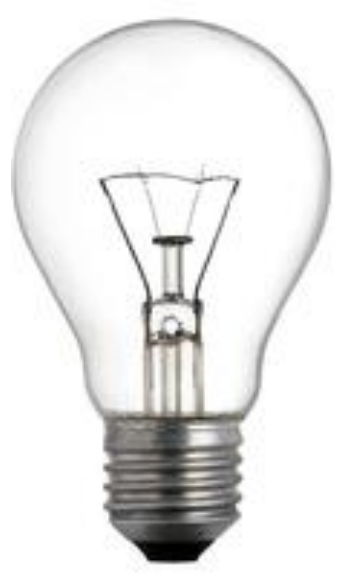

Intelligent Energy

Mangement

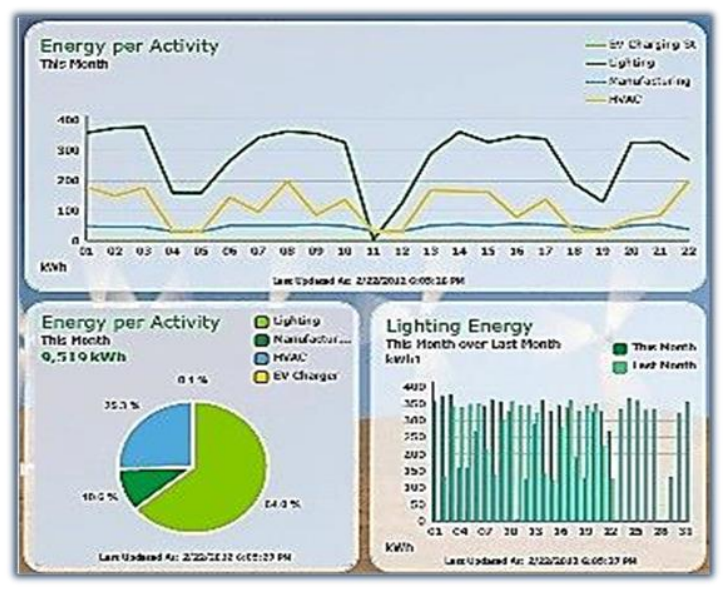

Schneider StruxureWare Energy Dashboard 


\section{Introduction}

\section{Three ways to improve Energy Efficiency in Buildings}

[Pérez-Lombard et al., 2008]

Energy

Conservation

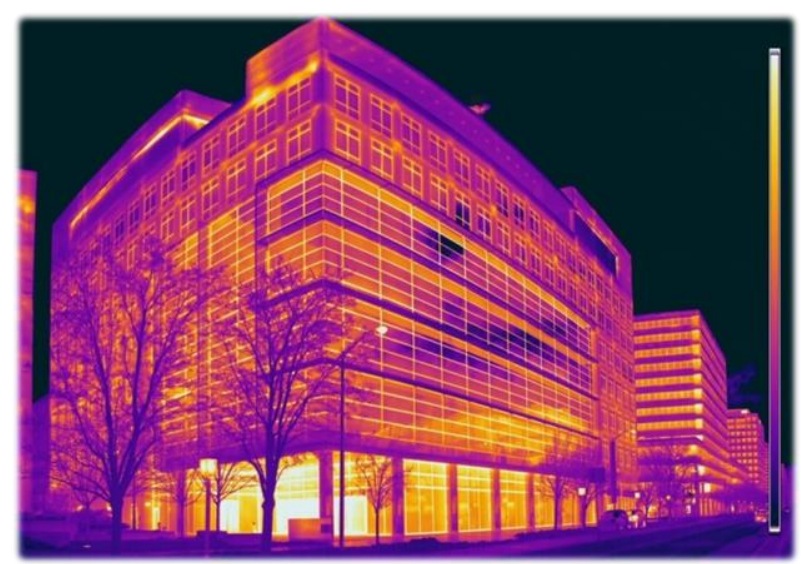

MIT Field Intelligence Laboratory
Equipment

Efficiency

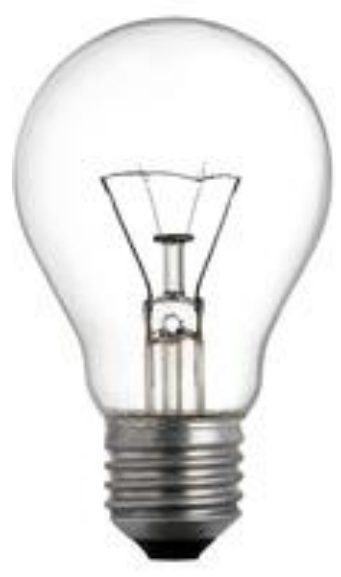

Intelligent Energy

Mangement

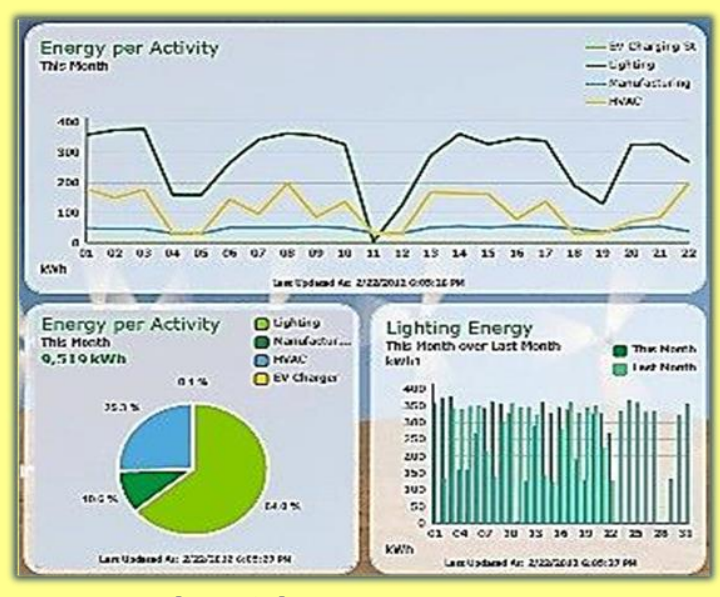

Schneider StruxureWare Energy Dashboard 


\section{Introduction}

\section{Intelligent Energy Management through an Energy Management System (EMS)}

\begin{tabular}{|c|c|c|c|}
\hline Building & \multicolumn{3}{|c|}{ Energy Management System (EMS) } \\
\hline $\begin{array}{l}\text { Energy } \\
\text { Metering } \\
\text { Network }\end{array}$ & $\begin{array}{l}\text { Monitors } \\
\text { Building } \\
\text { Energy } \\
\text { Consumption }\end{array}$ & and Upportunities & $\begin{array}{l}\text { Adjust Building } \\
\text { Energy } \\
\text { Management } \\
\text { Policies }\end{array}$ \\
\hline
\end{tabular}




\section{Introduction}

\section{An EMS track energy consumption in buildings to identify problems and misuse situations}

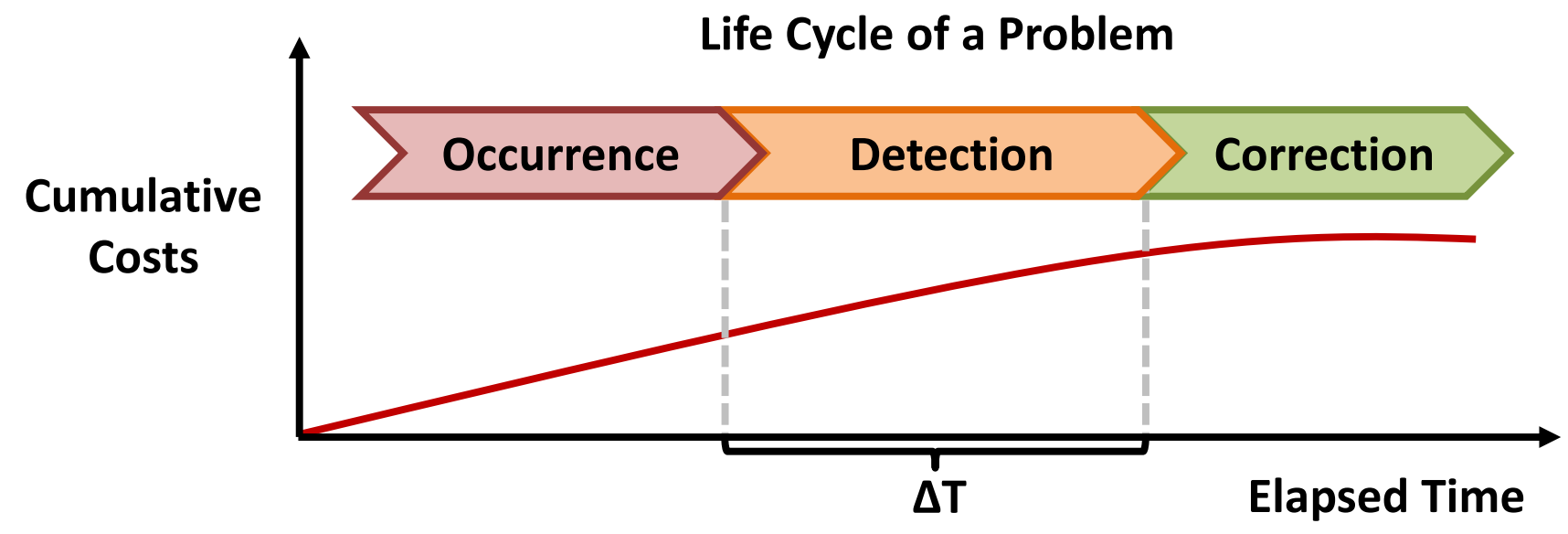




\section{Introduction}

An EMS track energy consumption in buildings to identify problems and misuse situations

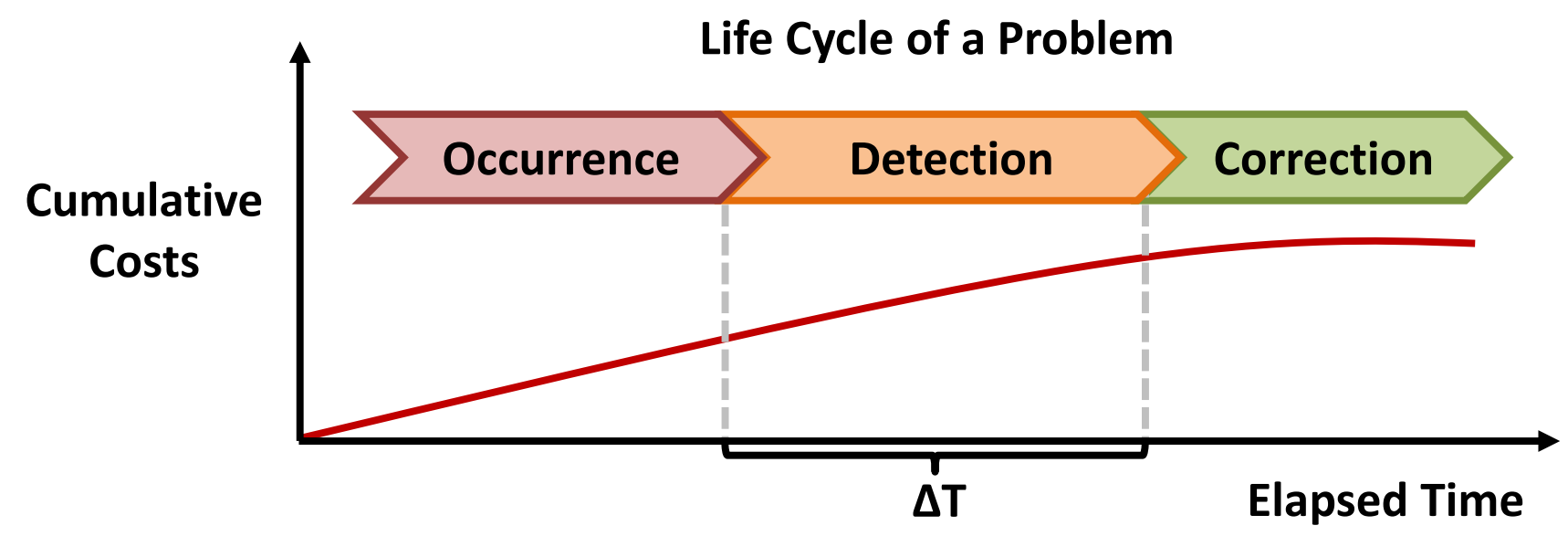

Effective Problem Solving
Early Problem Detection (small $\Delta \mathrm{T})$ 


\section{Introduction}

An EMS track energy consumption in buildings to identify problems and misuse situations

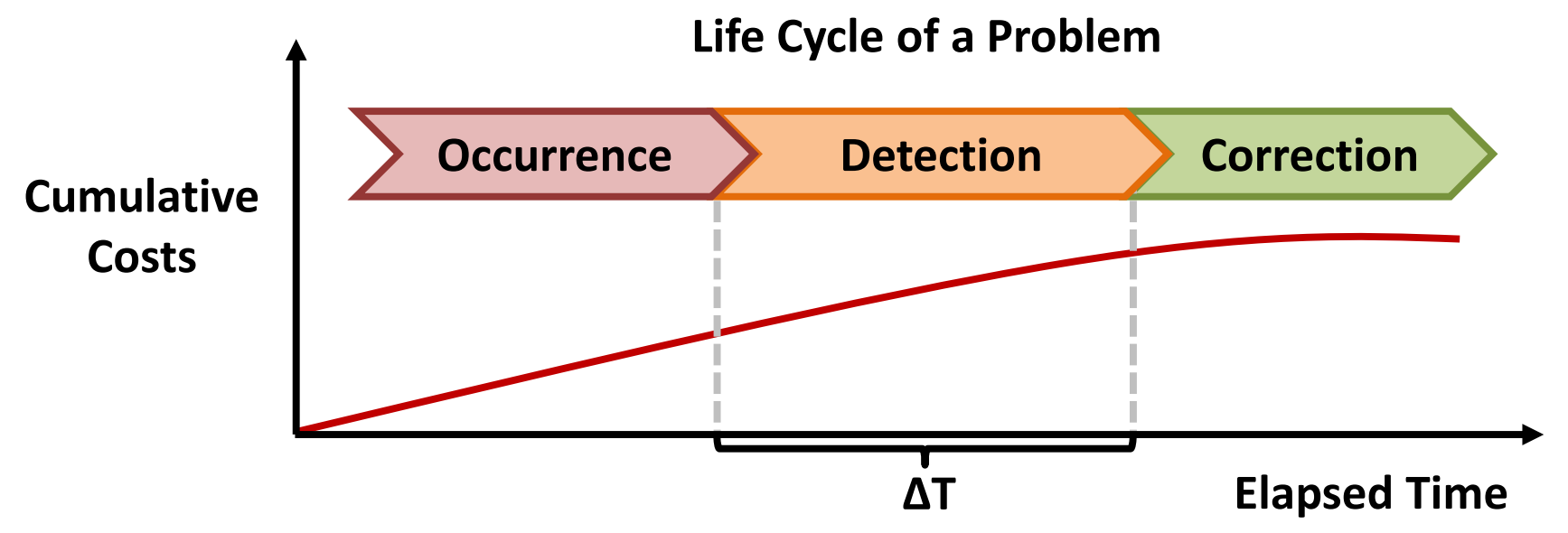

Data must be collected continuously and processed in (near) Real-Time 


\section{Agenda}

- Introduction

- State of the art

- Motivation

- Problem Statement

- Proposed Solution

- Evaluation

- Conclusion

- Bibliography 


\section{EMSs State of the art}

1. Ubiquitous Sensor Networks

- The raise of the "Internet of Things" (IOT)

2. Large sets of pervasive Sensor Data

- Leads to the challenges of Big Data 


\section{EMSs State of the art}

1. Ubiquitous Sensor Networks

- The raise of the "Internet of Things" (IOT)

2. Large sets of pervasive Sensor Data

- Leads to the challenges of Big Data

3. DBMSs are not capable of processing such sensor data in Real-Time [Golab and Özsu, 2003]

- Big Data is imposing a paradigm shift on how data is being processed 


\section{EMSs State of the art}

1. Ubiquitous Sensor Networks

- The raise of the "Internet of Things" (IOT)

2. Large sets of pervasive Sensor Data

- Leads to the challenges of Big Data

3. DBMSs are not capable of processing such sensor data in Real-Time [Golab and Özsu, 2003]

- Big Data is imposing a paradigm shift on how data is being processed

4. EMSs are supported by DBMSs [Kazmi et al., 2014]

-EMSs are not prepared to process sensor data in real-time [Anjos et al. 2014] 


\section{EMSs State of the art}

An EMS responding in Real-Time must be capable of process energy metering data within a deadline of 5 minutes [Granderson et al.,2011]

EMS

EEMSuite $^{1}$

EnergyWitness ${ }^{2}$

EnerwiseEM ${ }^{3}$

OEIS $^{4}$
Data Processing Infrastructure

DBMS

DBMS

DBMS

DBMS
Real-Time Monitoring

Hourly

Quarter Hour

Hourly

Hourly
1 - "Enterprise Energy Management Suite"

2 - "EnergyWitness"

3 - "Enerwise Energy Manager"

4 - "Open Energy Information System"
(McKinstry)

(Interval Data Systems, Inc.)

(Enerwise Global Tech.)

(Lawrence Berkeley National Lab.) 


\section{EMSs State of the art}

- EMSs relly on DBMSs to process energy metering data streams

- The latency of the persistence step is not compatible with the requirement of processing data in real-time

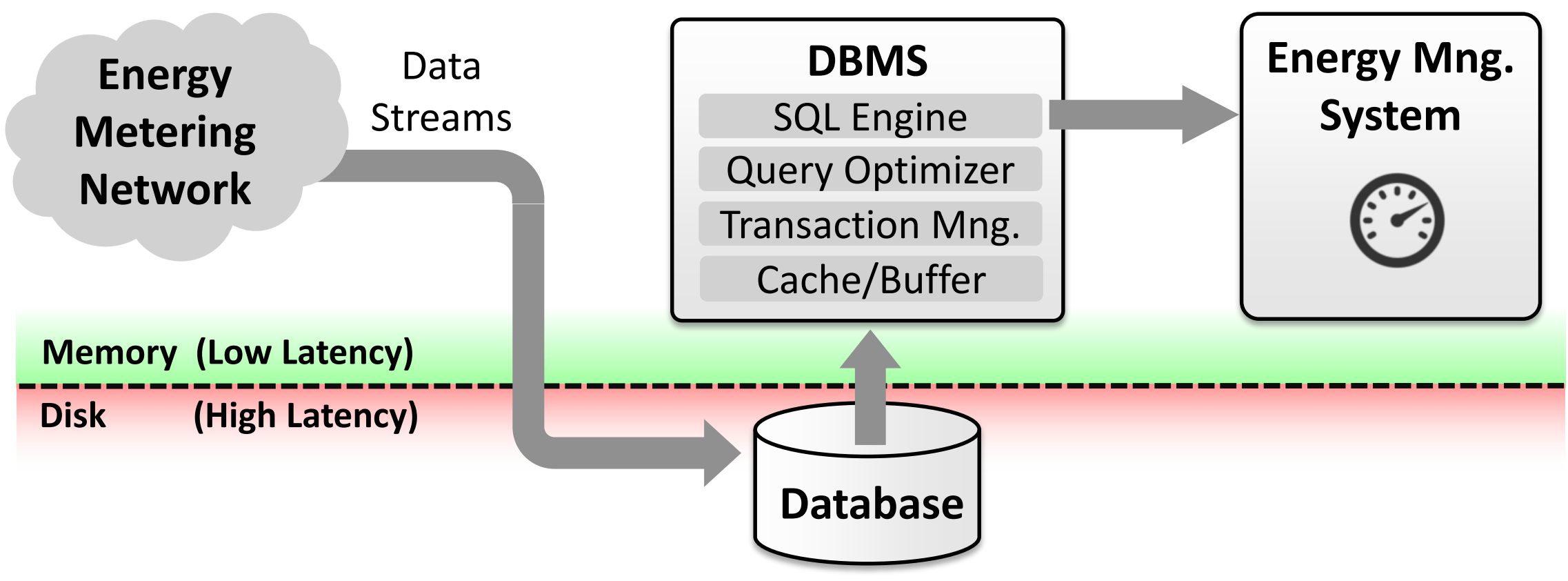




\section{State of the art}

There is some monitoring applications capable of real-time data processing, supporting a real-time decision making process

\section{Real-Time Monitoring Applications}

\section{Application Domain}

Stock Market Transactions

Healthcare Monitoring

Network Traffic

Weather Monitoring

\section{Authors}

(Chandramouli et al., 2010) (Mukherjee et al., 2010)

(Jiang et al., 2011) (Zhang et al., 2010)

(Akhtar and Siddiqui, 2011) 


\section{State of the art}

\section{We survey the architecture of these applications to conclude that they are supported by a DSMS (instead of by a DBMS)}

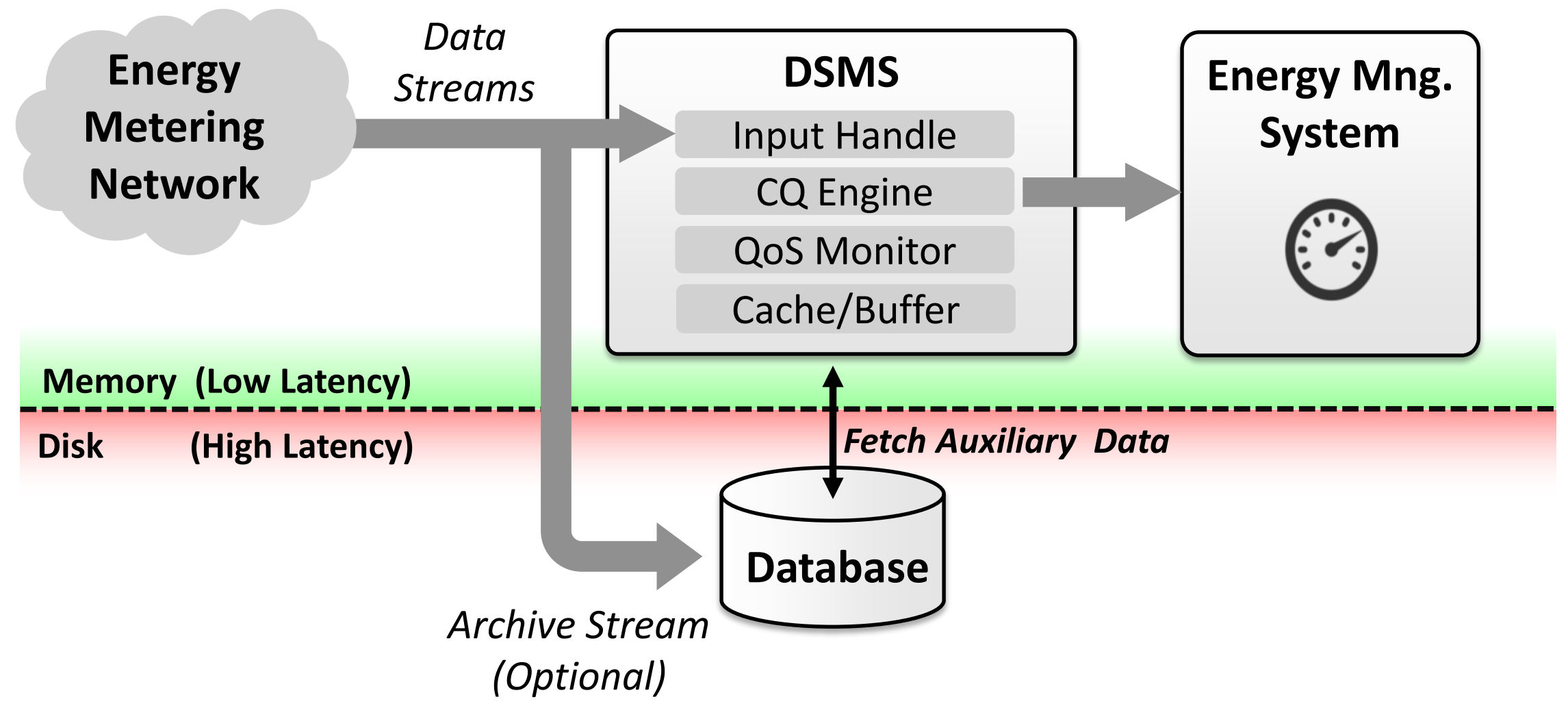




\section{State of the art}

Conclusions:

1. The generic architecture of an EMS is supported by traditional DBMSs (preventing RT data processing)

2. Sensor Network Monitoring Applications supported by DSMSs are capable of RT data processing 


\section{Agenda}

- Introduction

- State of the art

- Motivation

- Problem Statement

- Proposed Solution

- Evaluation

- Conclusion

- Bibliography 


\section{Motivation - Case Study}

Q1: Outliers. Identify the energy meters reporting an energy consumption $10 \%$ above the respective average over the last 8 hrs.

Q2: Fault Detection. Identify faulty meters that are not being able of produce energy consumption measurements every $60 \pm 5$ secs.

Q3: Variation. For each meter, determine the variation (\%) of energy consumption from the average over the last 5 mins.

Q4: Forecast. For each meter, give me its current and expected energy consumption. Being the expected value of each meter given by the AVG consumption of the current hour, computed along last month. 


\section{Motivation}

\section{Evaluating Query Q1}

Q1: "Identify the energy meters reporting an energy consumption $10 \%$ above the respective average over the last 8 hours."

\section{Energy Consumption Sensor Data}

Q1 Illustrative Evaluation

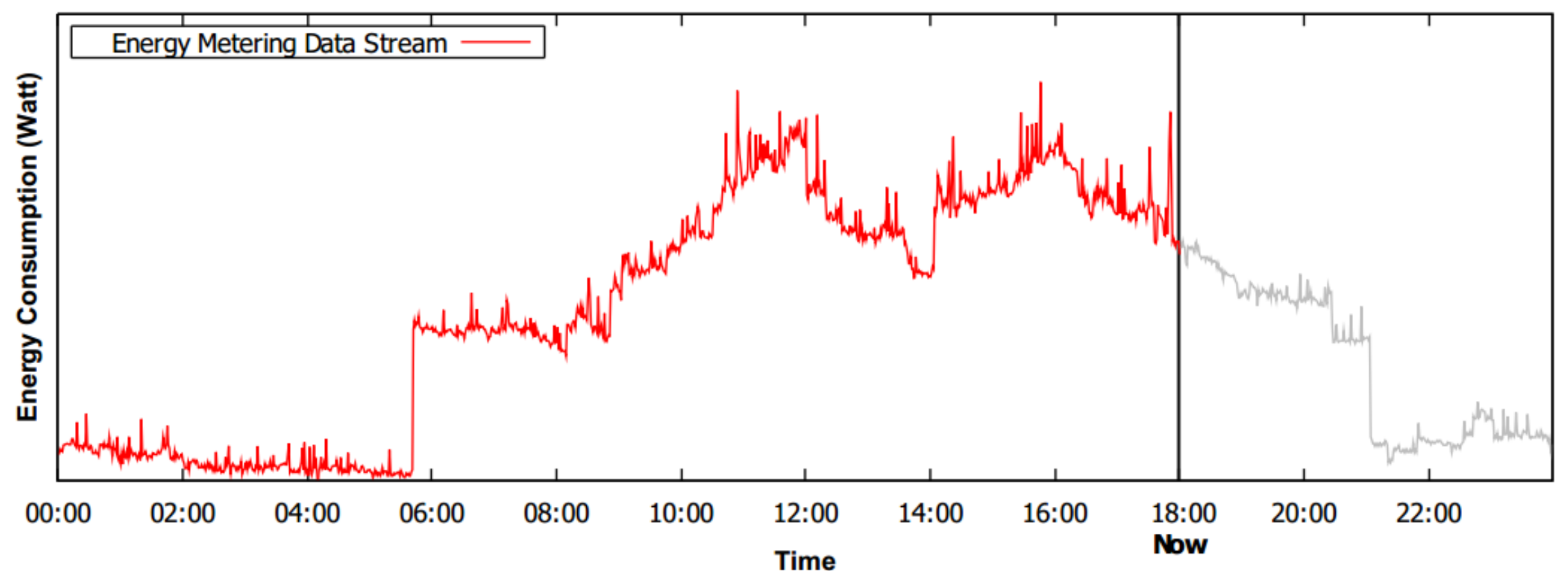


Evaluation Requirements of Query Q1:

I. Query must be Evaluated Continuously (the last-8h-from-now interval is changing continuously) 


\section{Motivation}

Evaluation Requirements of Query Q1:

I. Query must be Evaluated Continuously (the last-8h-from-now interval is changing continuously)

II. Low Query Evaluation Latency, in order to be possible to timely process new data as fast as it arrives-i.e. in Real-Time 


\section{Motivation}

Evaluation Requirements of Query Q1:

I. Query must be Evaluated Continuously (the last-8h-from-now interval is changing continuously)

II. Low Query Evaluation Latency, in order to be possible to timely process new data as fast as it arrives-i.e. in Real-Time

III. Compute Data Aggregates over Moving Windows (e.g. the AVG operator over the 8-hour sliding time window) 


\section{Motivation}

Suitability of each Query Engine to evaluate Query Q1:

\section{Requirements}

Queries Evaluated

Continuously

Low Query

Evaluation Latency

Data Aggregates over

Moving Windows
DBMS

One-Time

Queries

Disk Access

Overhead

Lacks
DSMS

Continuous

Queries

In-memory

Processing

Built-in 
Motivation

Suitability of each Query Engine to evaluate Query Q1:

\section{Conclusion}

\section{DBMSs are not the best solution to} support a Real-Time Monitoring EMS 


\section{Agenda}

- Introduction

- State of the art

- Motivation

- Problem Statement

- Proposed Solution

- Evaluation

- Conclusion

- Bibliography 


\section{Problem Statement}

This dissertation aims to validate the thesis that:

"An EMS supported by a DSMS outperforms an EMS supported by a DBMS, on processing energy metering data in real-time."

\begin{tabular}{c|l} 
Better & i. Lower Query Evaluation Latency \\
Performance & ii. Greater Query Language Expressibility
\end{tabular} 


\section{Agenda}

- Introduction

- State of the art

- Motivation

- Problem Statement

- Proposed Solution

- Evaluation

- Conclusion

- Bibliography 


\section{Proposed Solution}

Proposed Data Processing Architecture

Generic Architecture of an EMS is organized in $\mathbf{3}$ Tiers 


\section{Proposed Solution}

\section{Proposed Data Processing Architecture}

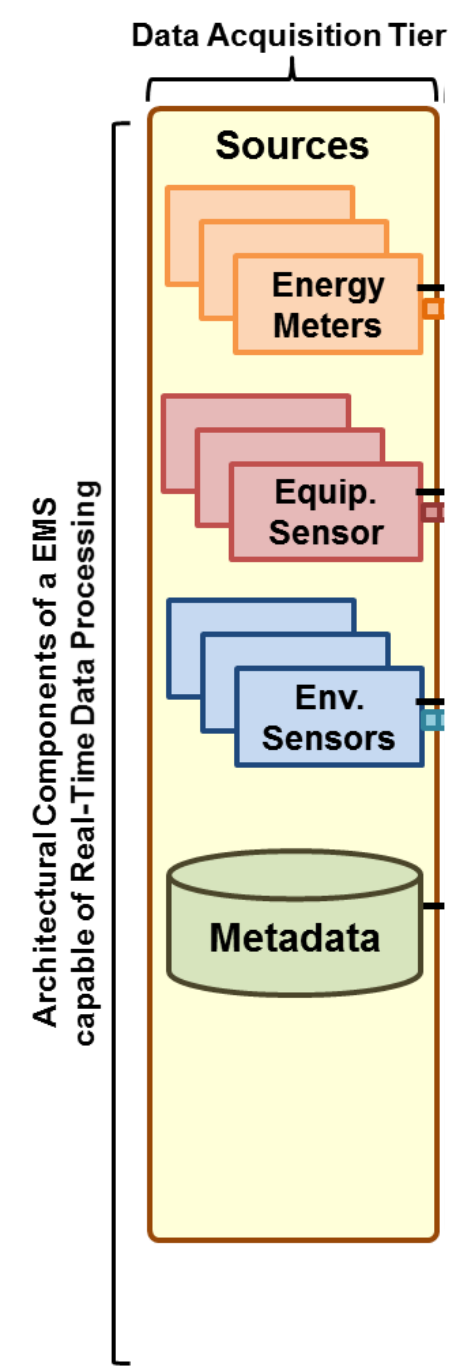




\section{Proposed Solution}

\section{Proposed Data Processing Architecture}
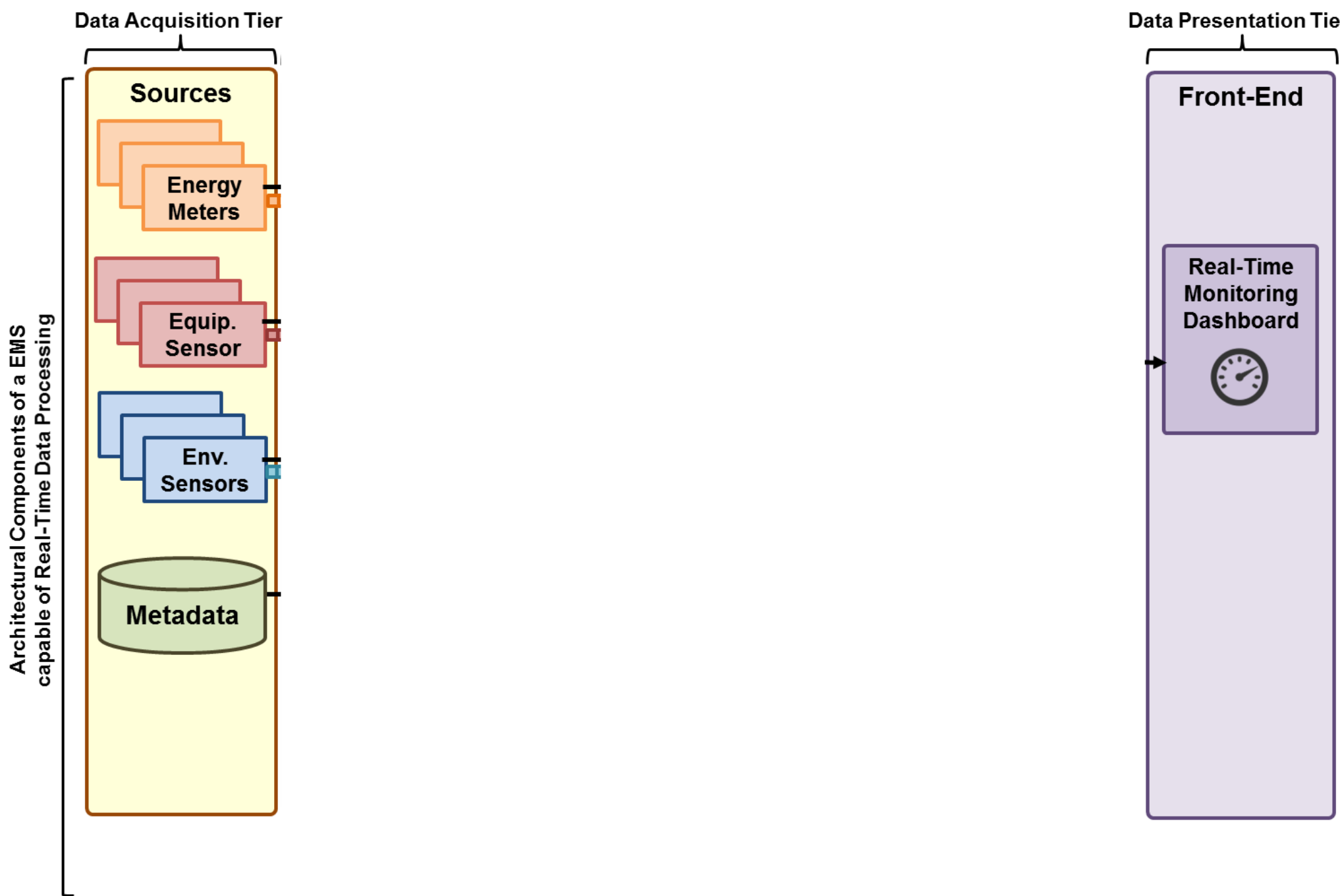


\section{Proposed Solution}

\section{Proposed Data Processing Architecture}

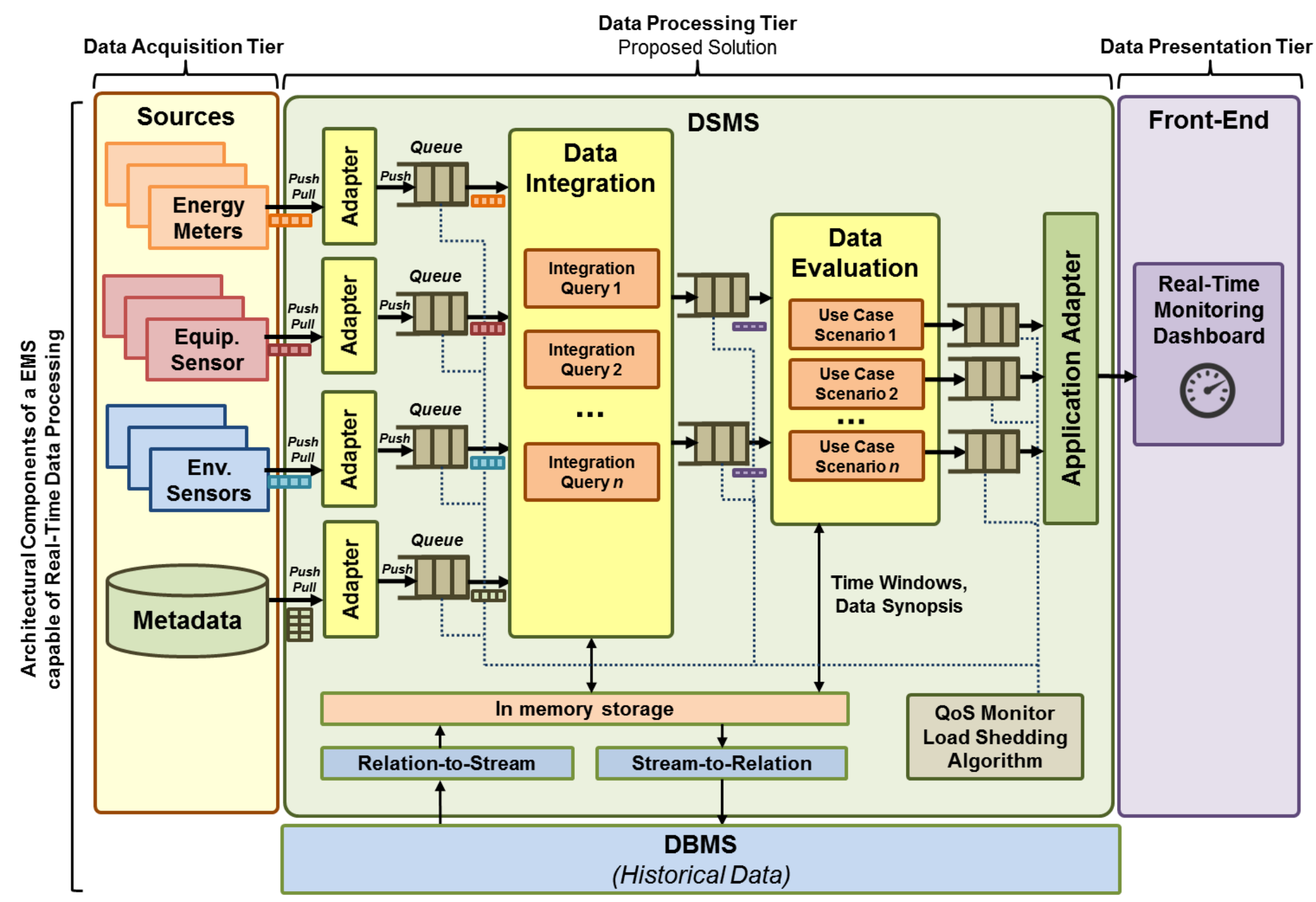




\section{Proposed Solution}

\section{Proposed Data Processing Architecture}

\section{Proposed Architecture main feature:}

- Pipeline of data transformations supported by a DSMS

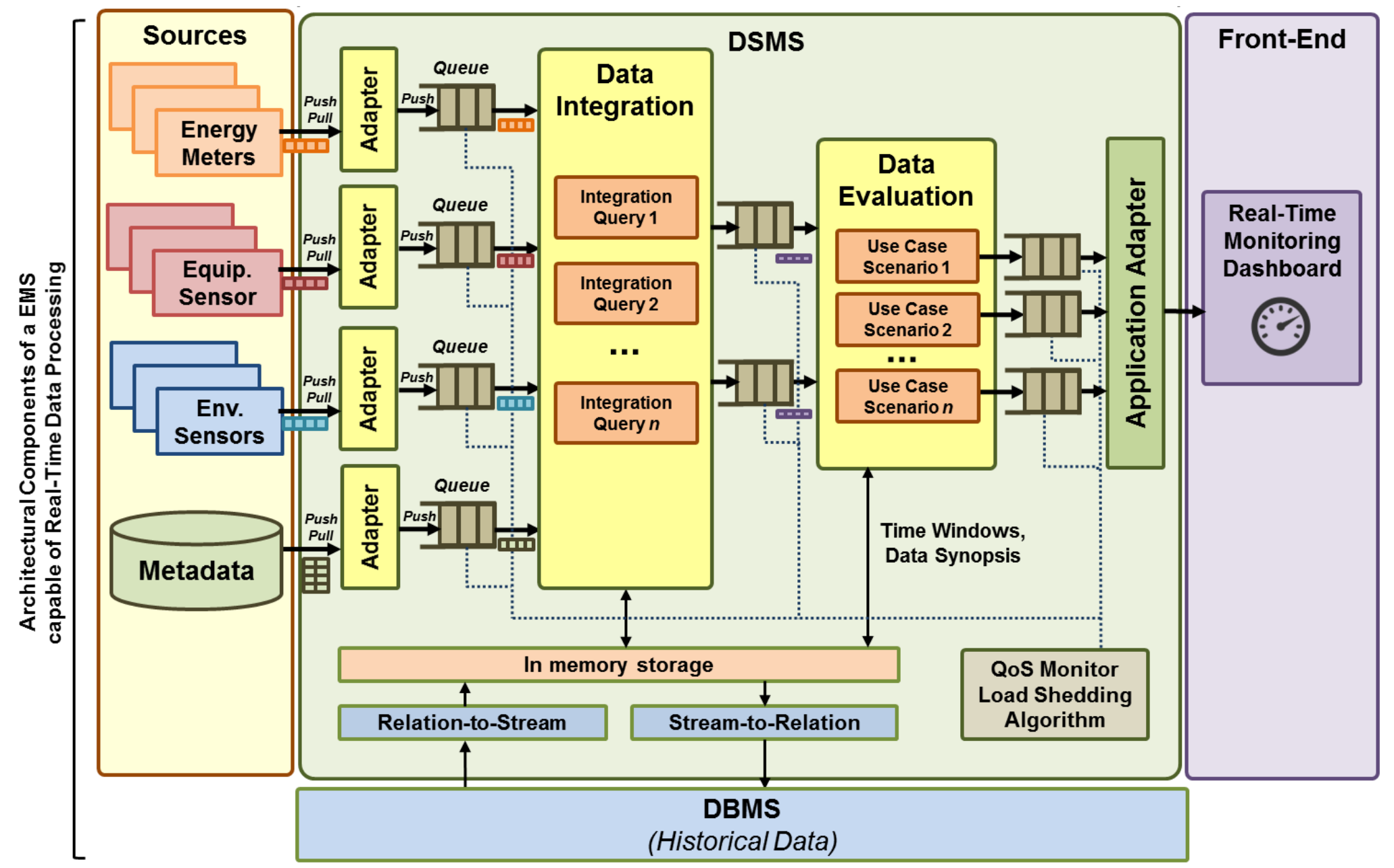




\section{Proposed Solution}

\section{Proposed Data Processing Architecture}

Adapters. Mediate the extraction of data from several sources into the data transformation process.

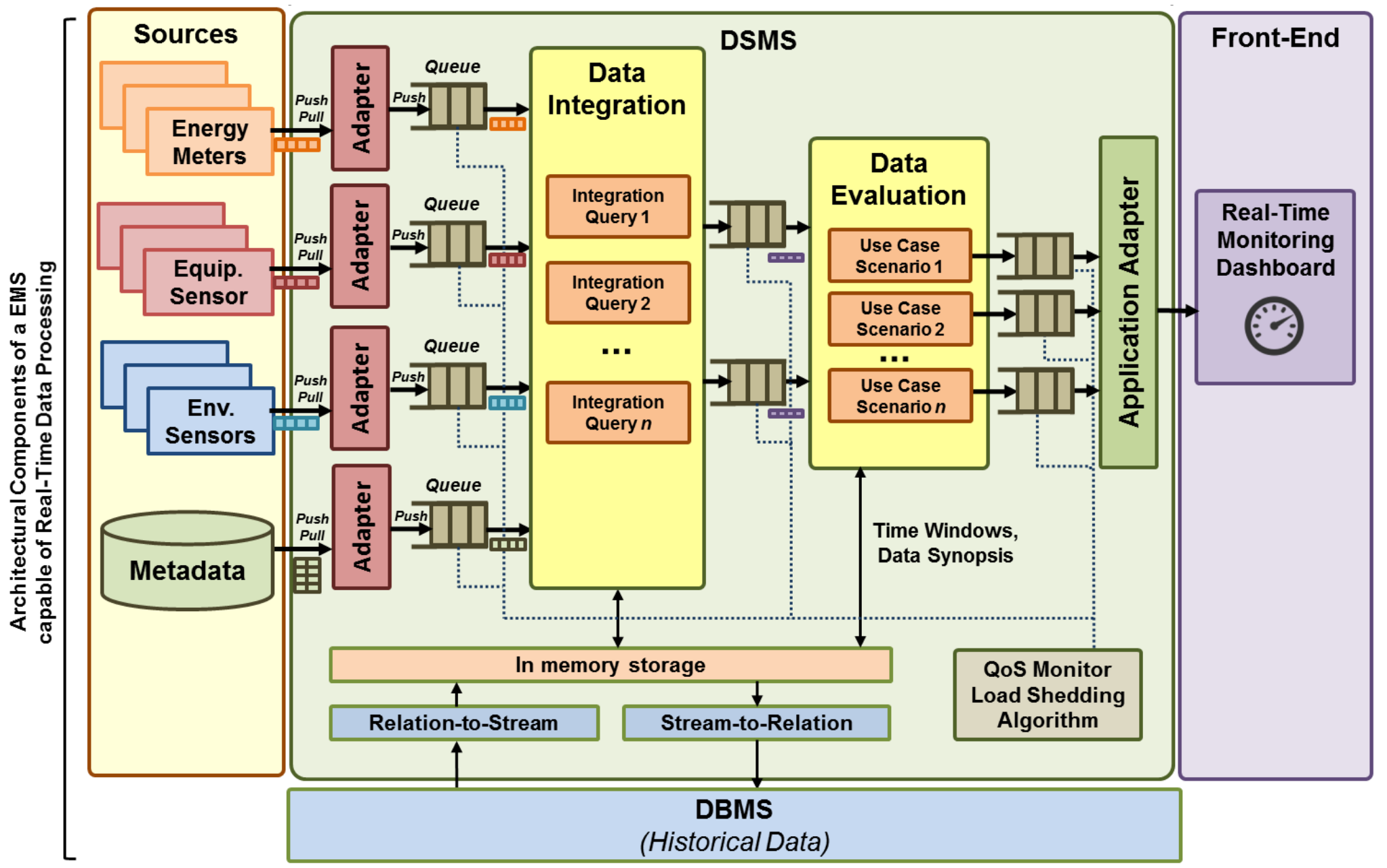




\section{Proposed Solution}

\section{Proposed Data Processing Architecture}

Data Integration. Data cleaning and integration steps used to compute a set of data streams tightly related with the problem domain.

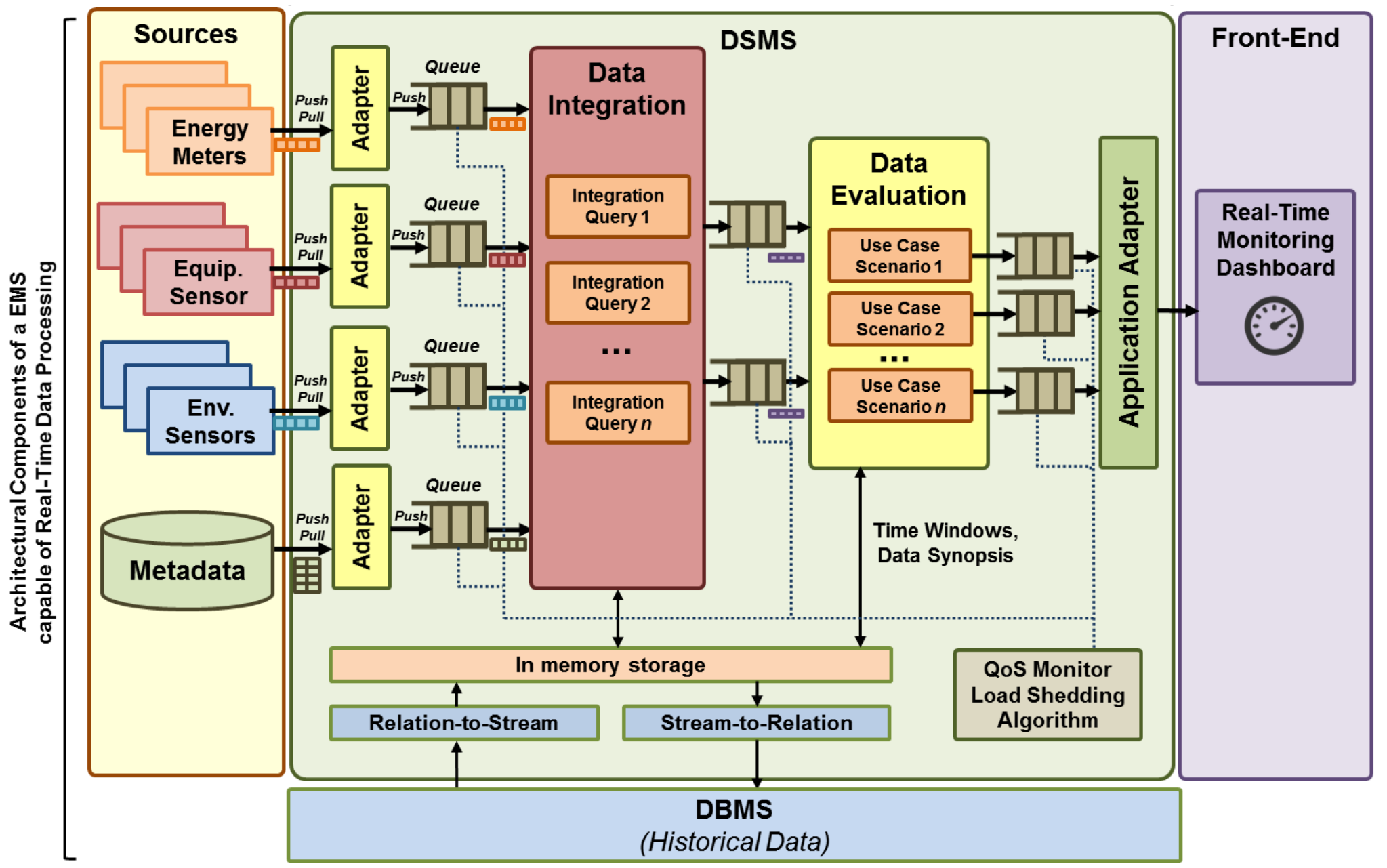




\section{Proposed Solution}

\section{Proposed Data Processing Architecture}

Data Evaluation. Supports the evaluation of energy management queries, those representing the energy monitoring use-case scenarios.

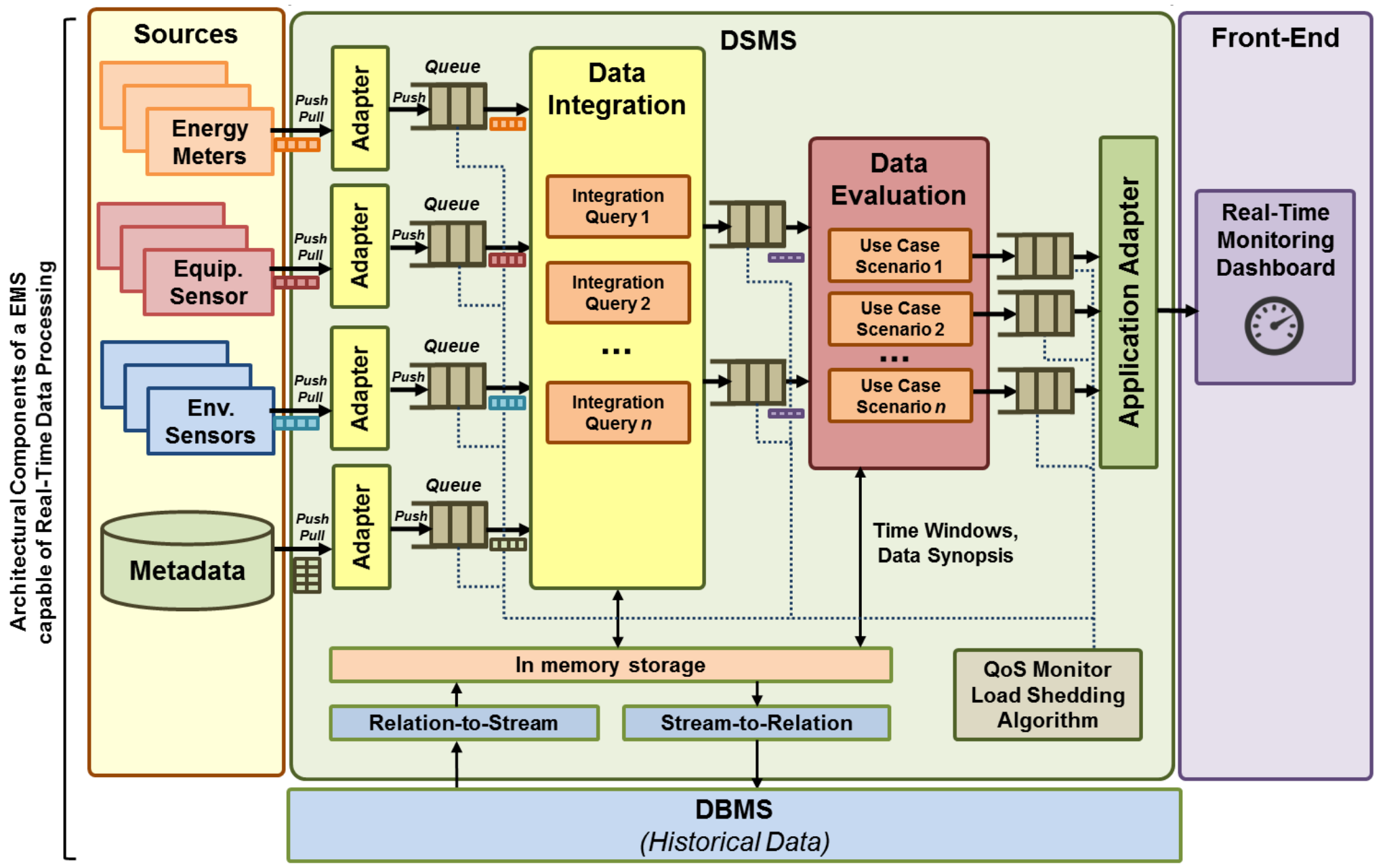




\section{Proposed Solution}

Requirements Analysis

\section{Literature Survey to identify the type of Data Transformations (case study) that must be performed by the proposed Data Processing Arch.}

\section{Surveyed Authors}

- Akhtar and Siddiqui, 2011

- Babcock et al., 2002

- Bizer et al., 2009

- Bonnet et al., 2001

- Chandramouli et al., 2010

- Cranor et al., 2003

- Madden and Franklin, 2002

- Mukherjee et al., 2010

- Zhang et al., 2010

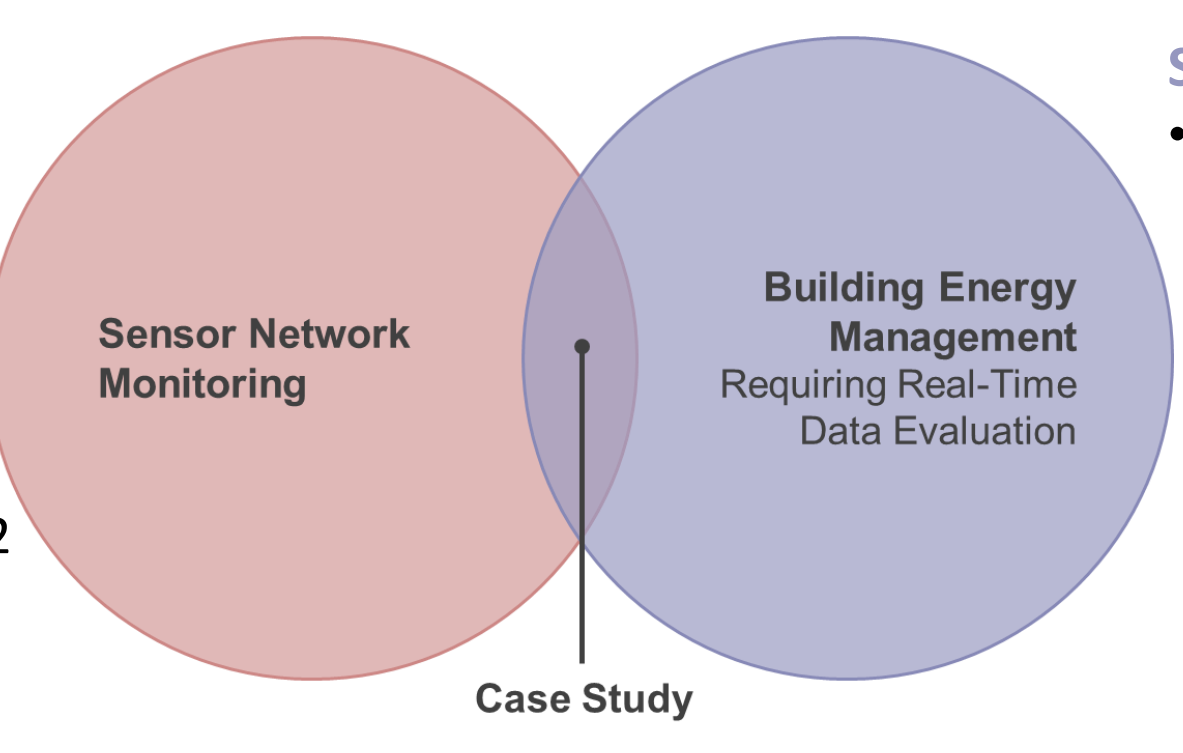

Surveyed Authors

- Granderson et al., 2011 


\section{Proposed Solution}

\section{Requirements Analysis}

\section{Case Study: 16 queries covering the surveyed application domains}

\begin{abstract}
Q\#

\section{Use Case Scenarios}

1 Identify the energy meters for which the reported energy consumption variation, along the last 5 minutes, has increased more than $20 \%$.

2 Identify the energy meters producing measurements with a periodicity out of $60+-5$ range of seconds.

$3^{\dagger}$ Identify the energy meters that are reporting energy consumption measurements $20 \%$ above than the respective average of last 24 hours.

4 For each energy meter, computes the fraction of its reported measurements relative to the total of energy that is being consumed by the building.

5 Sort in decreasing order the energy meters by its current energy consumption 5 measurements.

$6^{+}$For each energy meter and building as a whole, compute the Minimum/Maximum energy consumption ratio along last hour.

7 Identify the energy meters that are reporting measurements above a respective threshold.
\end{abstract}

\begin{tabular}{cccc} 
& \multicolumn{3}{c}{ Class of Queries } \\
\hline Detection & Data & Data & \\
of & Stream & Stream & Database \\
Abnormal & Instant & Metadata & Integration \\
Values & $\begin{array}{c}\text { Summary } \\
\text { Mettrics }\end{array}$ & $\begin{array}{c}\text { Evaluation } \\
\end{array}$ & \\
\hline
\end{tabular}

$8^{+} \quad(\ldots)$

\section{Integration Queries to support Use Case Scenarios}

For each data stream, aggregate its three-phase current measurements into the

10 total amount of energy being consumed, and then adorn the result stream with the location properties of the respective energy meter, which is available through a database of metadata.

$11^{+}$For each data stream, compute the variation of its current measurement value from the moving average of last 5 minutes.

$12^{\dagger}$ For each data stream, compute the period between its two last measurements.

$13^{\dagger}(\ldots)$
Class of Queries

Technique

Load Peak Load Profiling Analysis
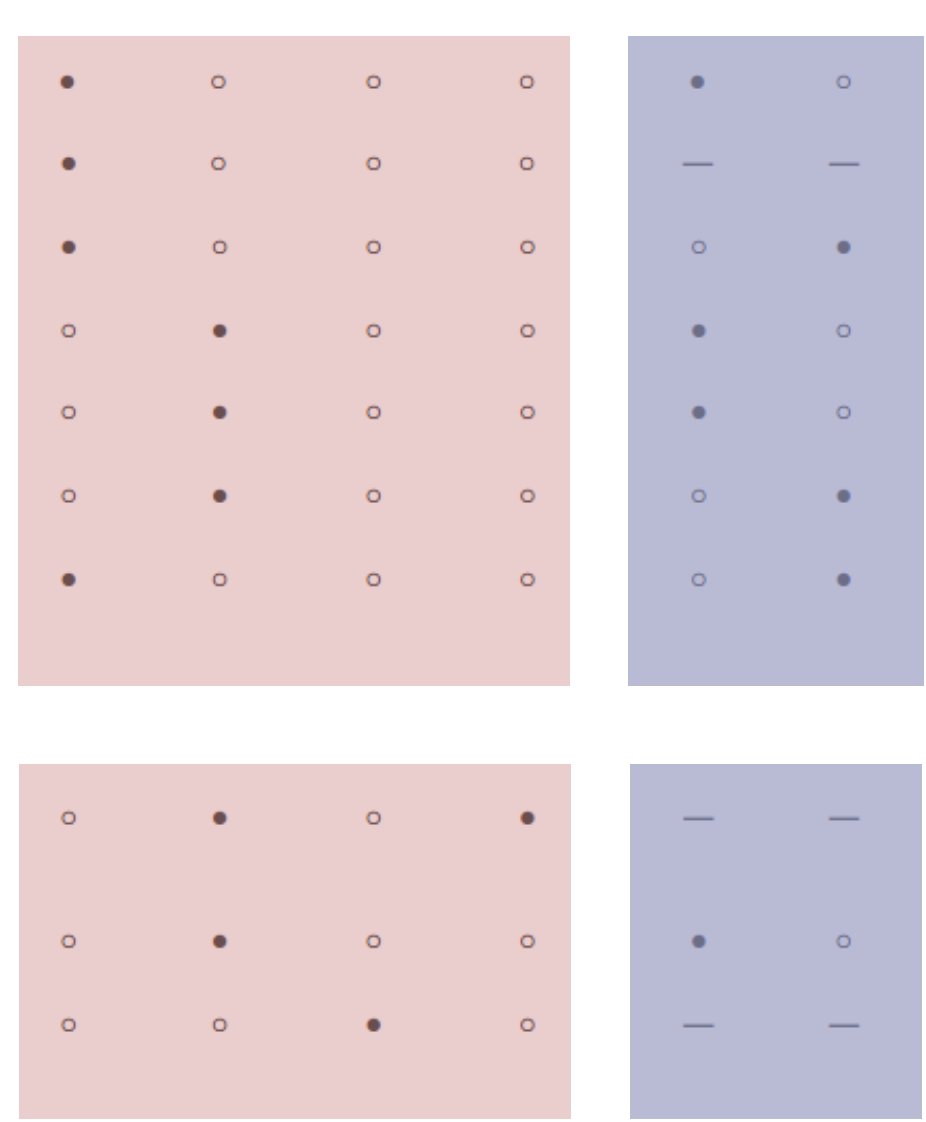


\section{Proposed Solution}

\section{Requirements Analysis}

Case Study: 9 Scenarios on monitoring building energy consumption, composed by a graph (pipeline) of 16 Queries

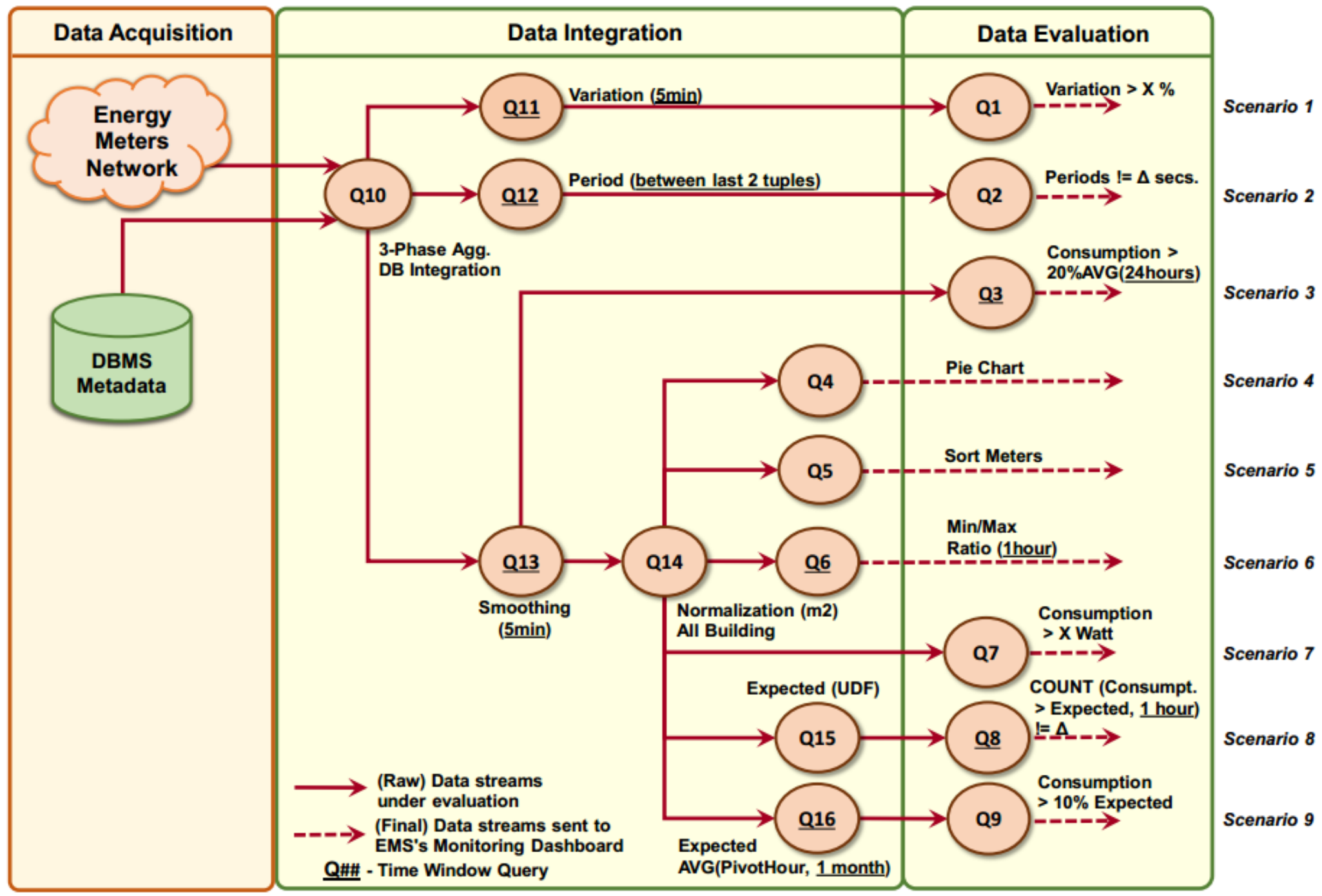




\section{Proposed Solution}

\section{Requirements Analysis}

Scenario 3: "Identify the energy meters reporting an energy consumption (Motivation Q1) 20\% above the respective average over the last 24 hours."

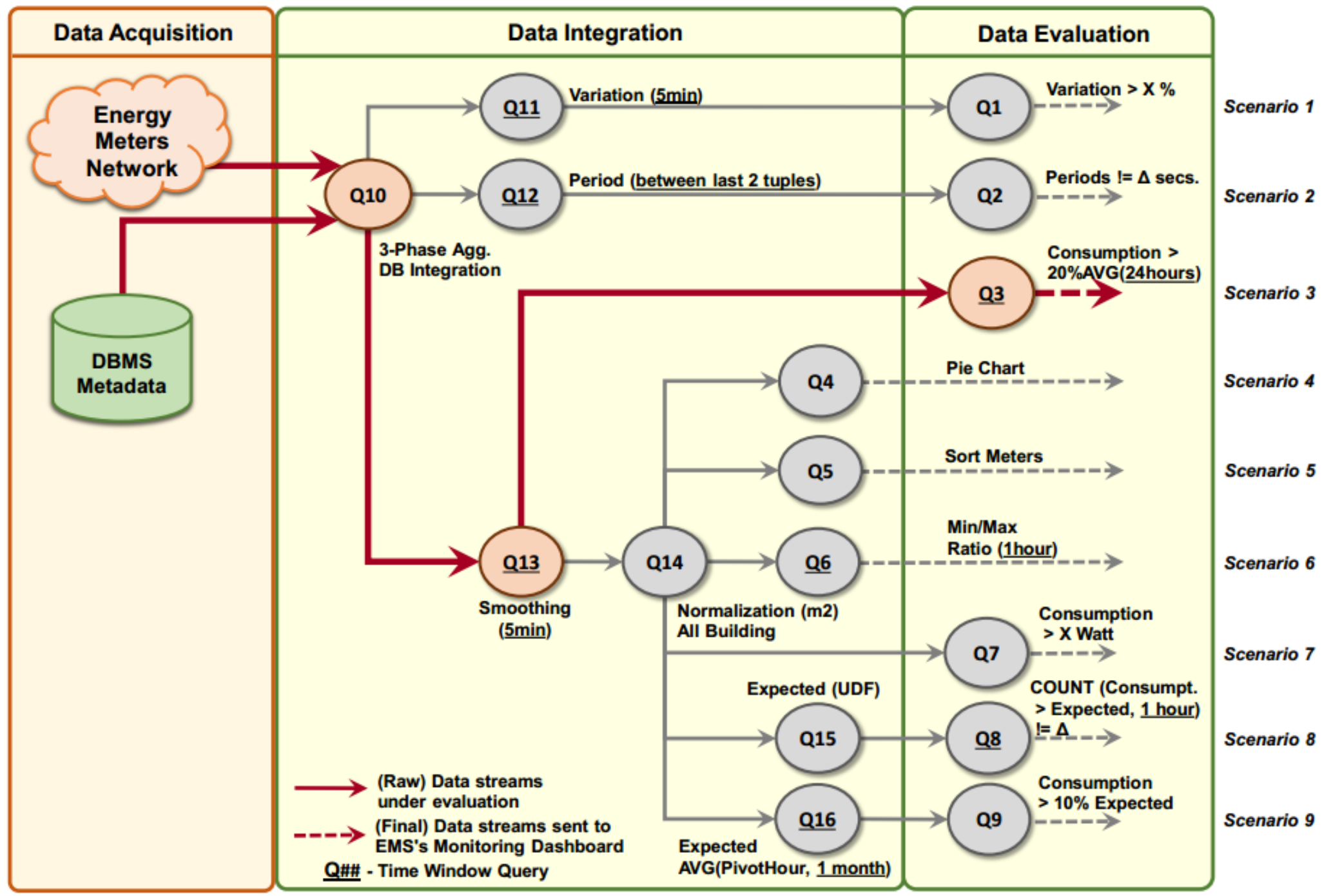




\section{Proposed Solution}

Case Study - Building Energy Metering Network

Instituto Superior Técnico

(4)

Taguspark Campus

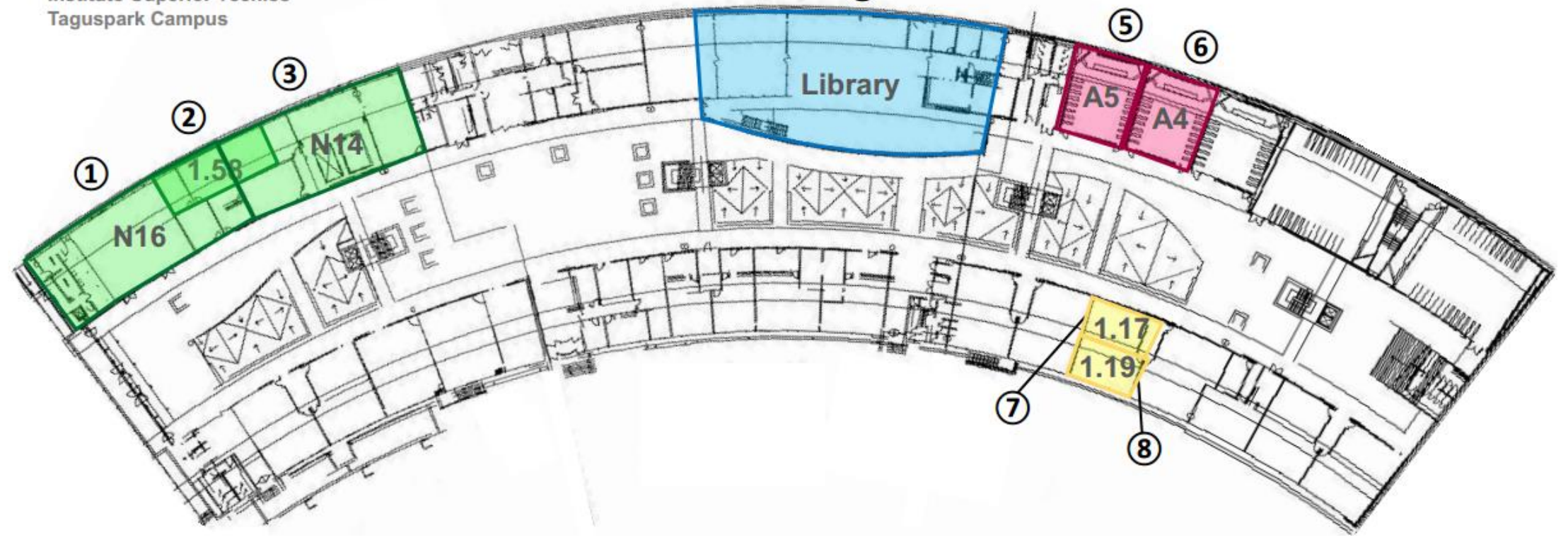

- Energy metering network of 8 meters

- 8 locations being continuously monitored $\left(\approx\right.$ total $\left.1365 \mathrm{~m}^{2}\right)$ (Four types of places: Offices, Library, Lecture Halls, and Class Rooms)

- Real Environment, Real Dataset

- 3-Phase Current power supplying the building 


\section{Agenda}

- Introduction

- State of the art

- Motivation

- Problem Statement

- Proposed Solution

- Evaluation

- Conclusion

- Bibliography 


\section{Evaluation}

\section{Methodology}

- Implementation of two prototypes of the proposed Data Processing Architecture:

1. DSMS supported (our proposed approach)

2. DBMS supported

(state-of -the-art approach)

Two ortogonal implementations of Proposed Data Processing Architecture

Benchmark Evaluation
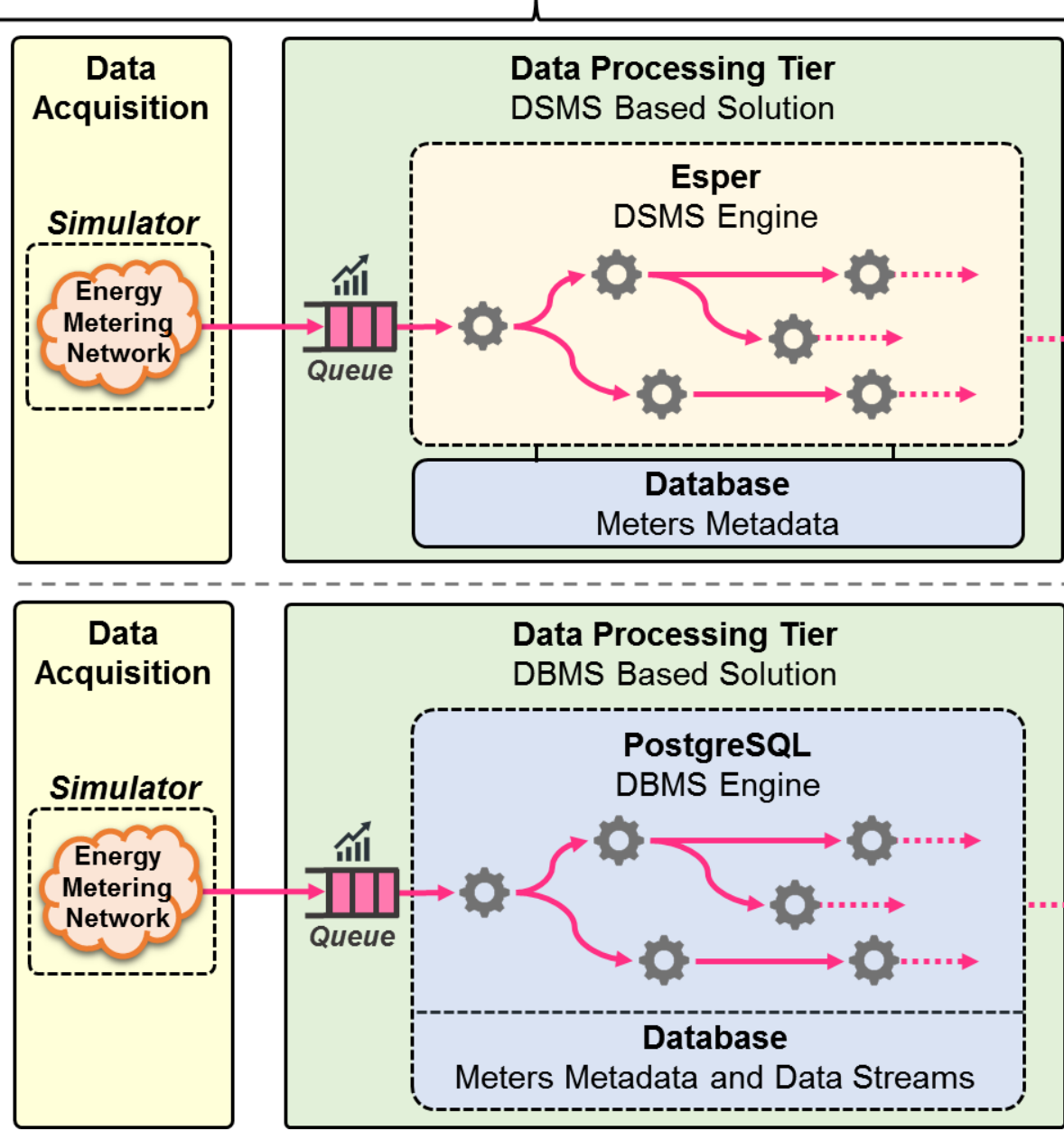


\section{Evaluation}

\section{Methodology}

Two ortogonal implementations of

Proposed Data Processing Architecture

Benchmark Evaluation

- Implementation of two prototypes of the proposed Data Processing Architecture:

1. DSMS supported (our proposed approach)

2. DBMS supported

(state-of -the-art approach)

- To perform a Benchmark Evaluation between the proposed solution and a state-of-the-art based solution

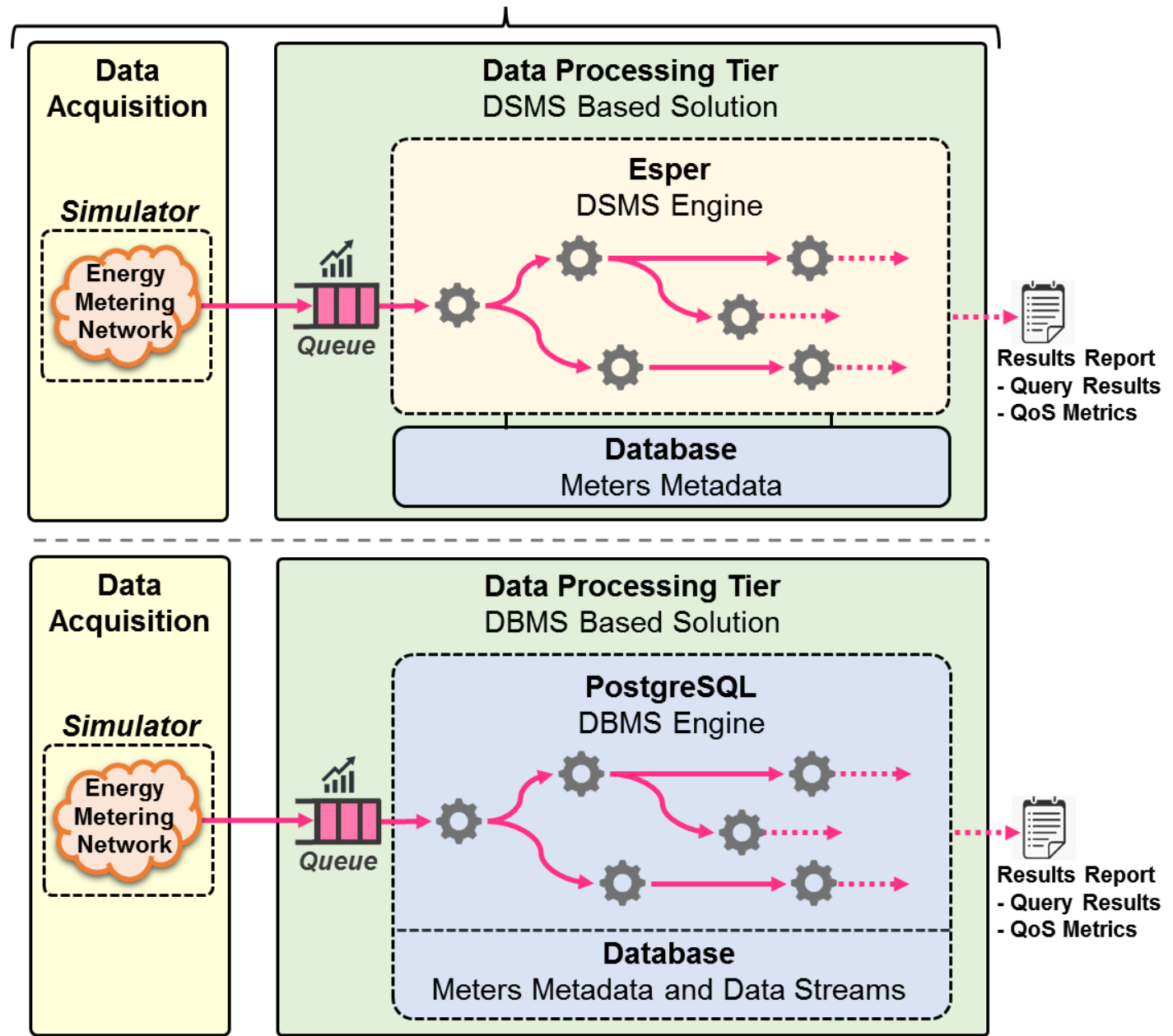




\section{Evaluation}

\section{Methodology}

Two ortogonal implementations of

Proposed Data Processing Architecture

Benchmark Evaluation

- DSMS Solution

supported by ESPER

i. An open-source DSMS that provides a declarative SQL-like query language

ii. Allowing a side-by-side comparison with DBMS's SQL
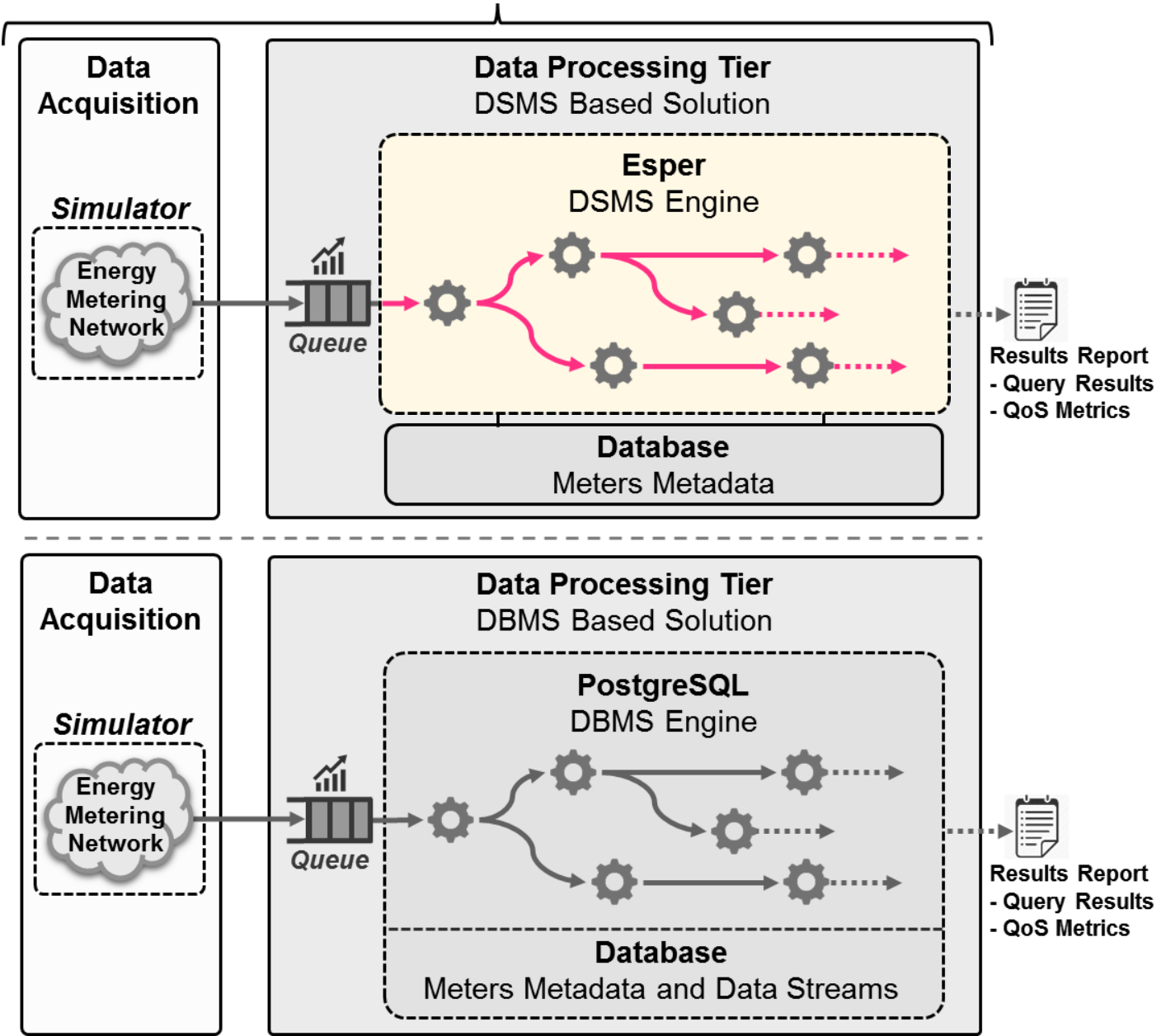


\section{Evaluation}

\section{Methodology}

Two ortogonal implementations of

Proposed Data Processing Architecture

Benchmark Evaluation

- DSMS Solution

supported by ESPER

i. An open-source DSMS that provides a declarative SQL-like query language

ii. Allowing a side-by-side comparison with DBMS's SQL

\section{- DBMS Solution}

\section{supported by PostgreSQL}

i. The most well-known and widely-used open-source DBMS
ـ
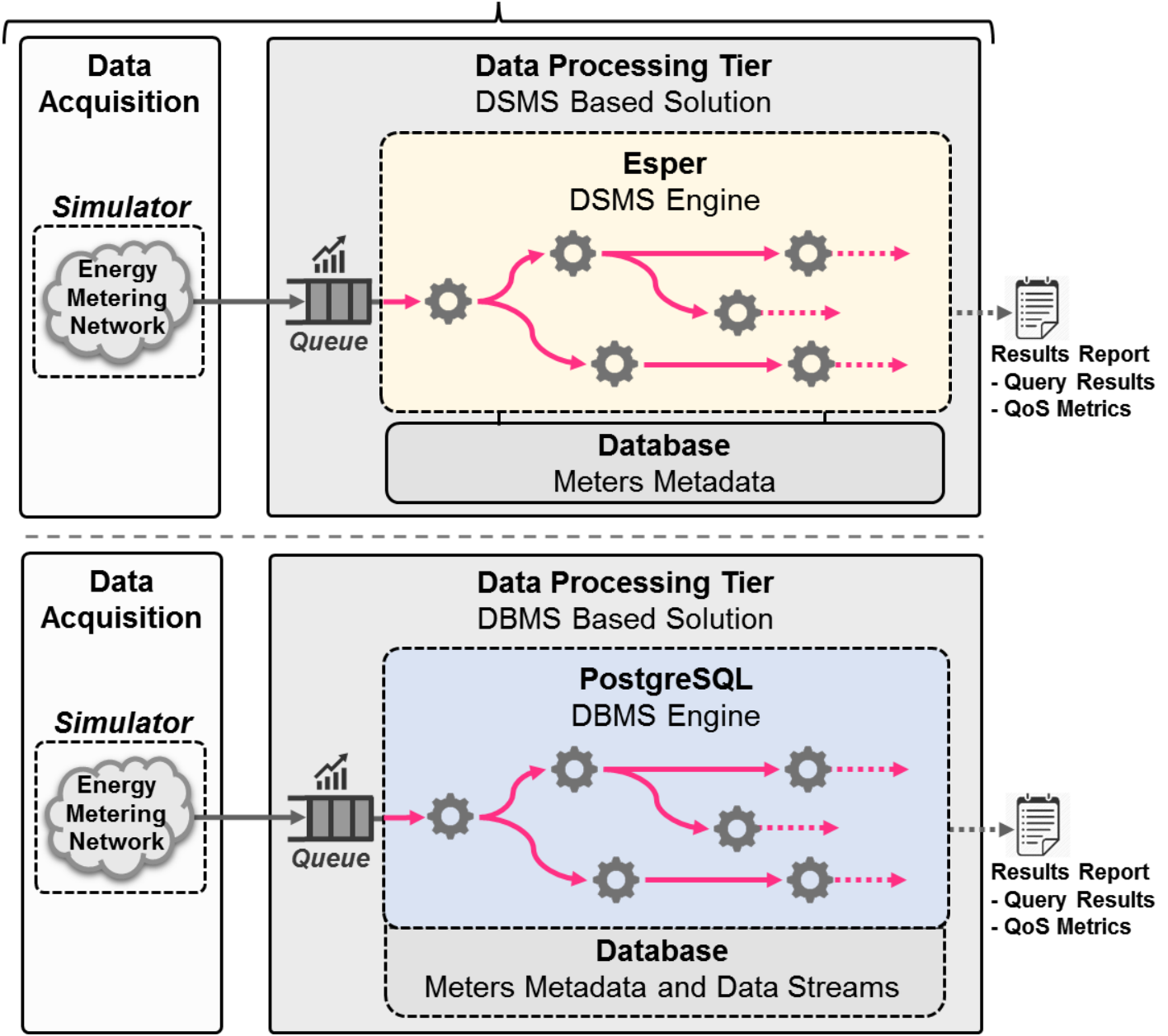


\section{Evaluation}

\section{Methodology}

Two ortogonal implementations of Proposed Data Processing Architecture

Benchmark Evaluation

\section{All 9 Scenarios \\ were implemented in both DSMS and DBMS solutions}

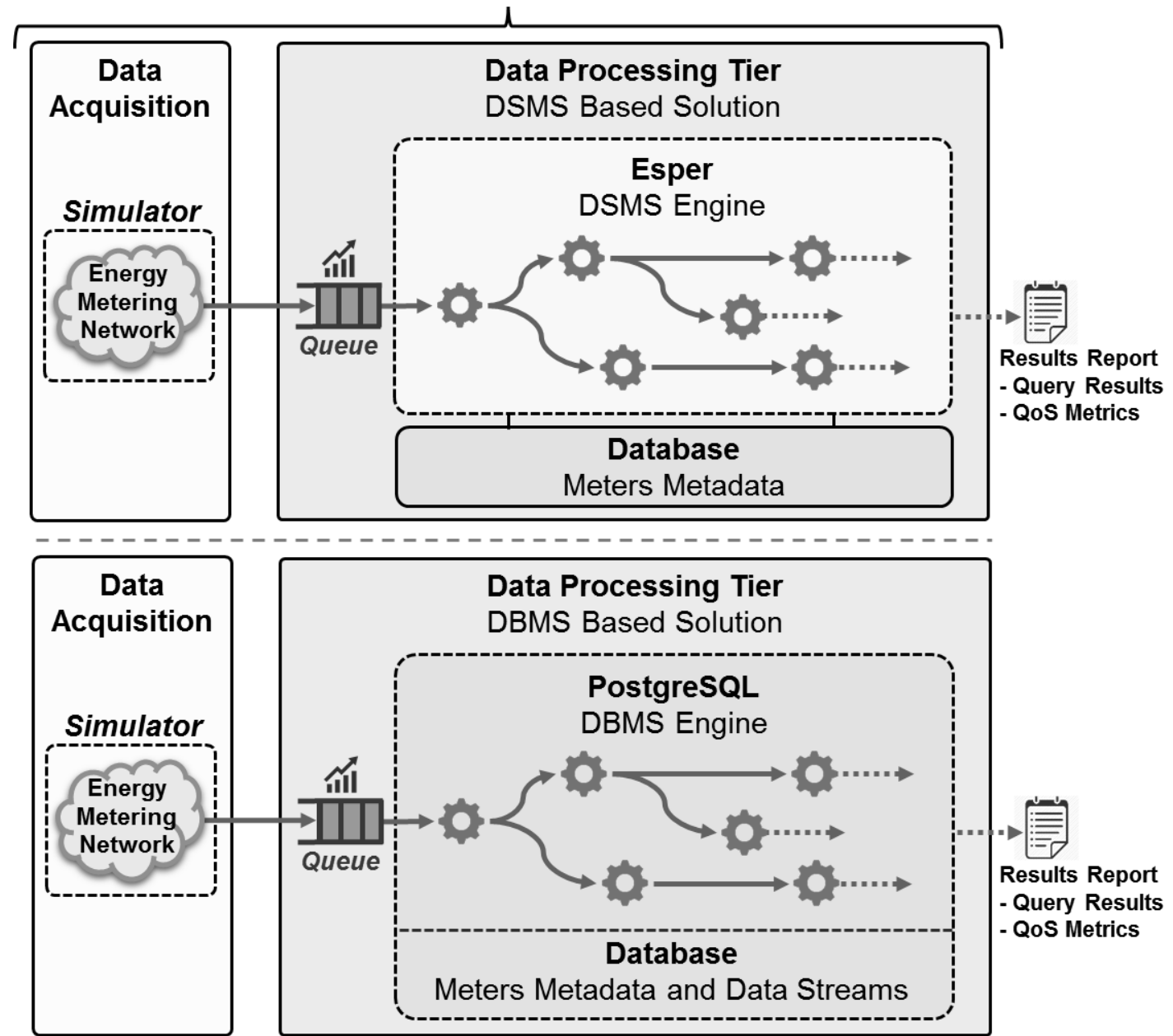




\section{Evaluation}

\section{Methodology}

Two ortogonal implementations of

Proposed Data Processing Architecture

Benchmark Evaluation
All 9 Scenarios
were implemented in both DSMS and DBMS solutions

- Evaluation Dimensions:

1. Query Language Evaluation

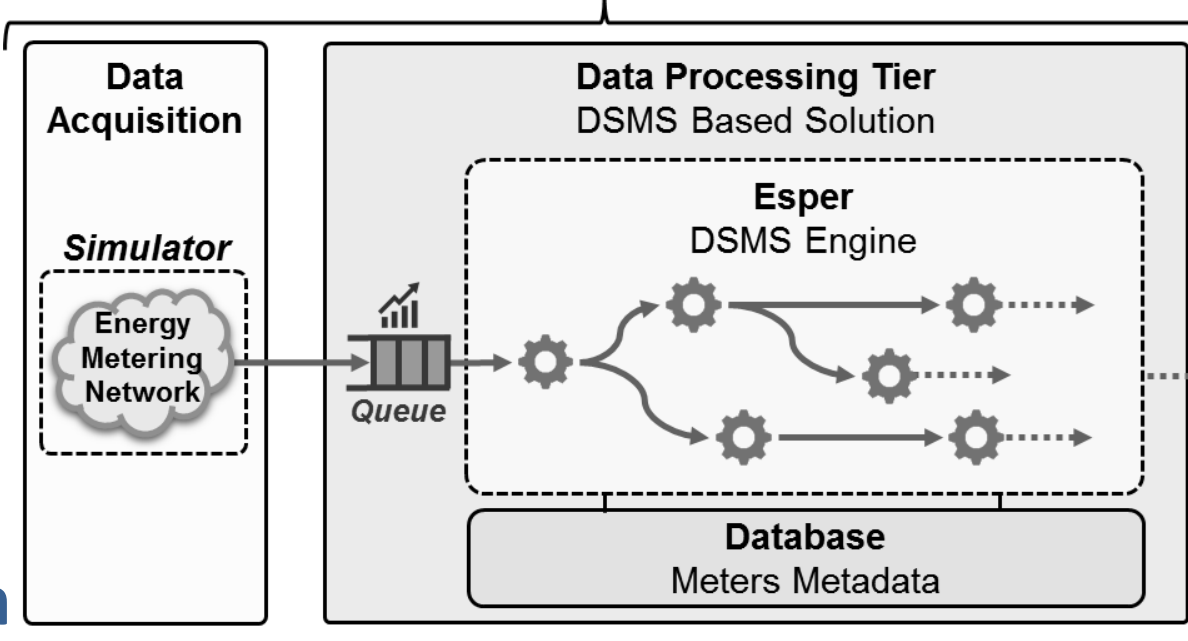

2. Performance Evaluation

- Assess the ability of each solution to express and timely evaluate the 9 Scenarios

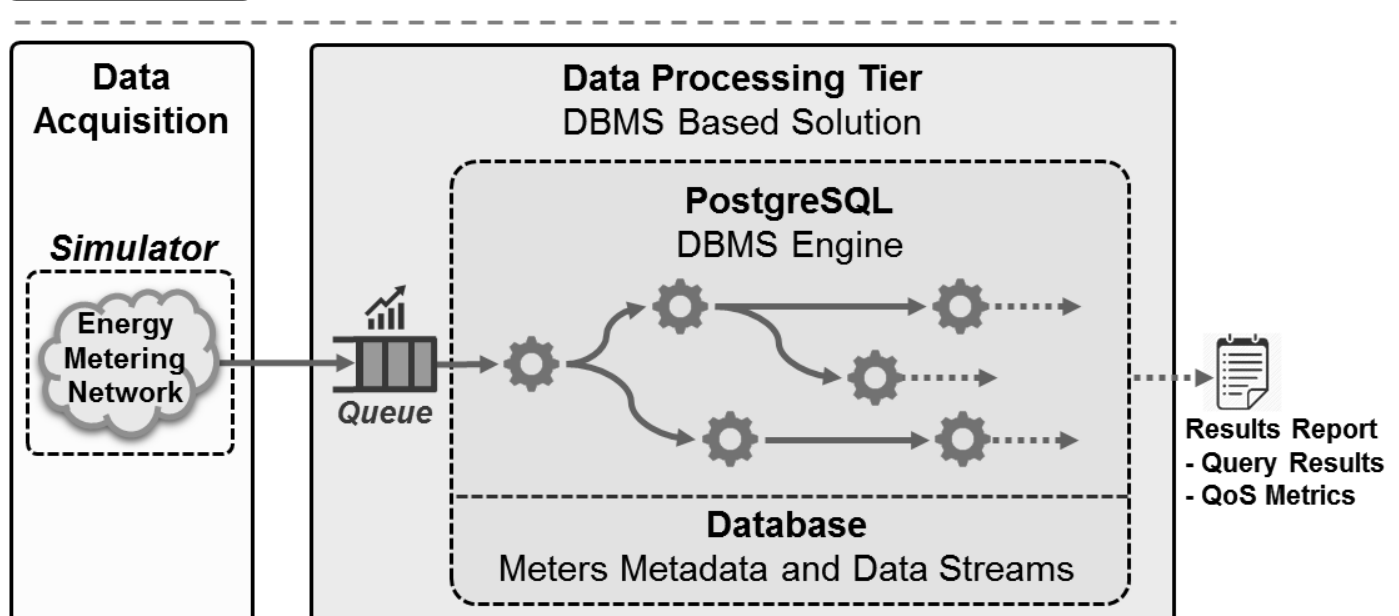




\section{Evaluation}

\section{Query Language Evaluation}

Relative difficulty of implementing the Use-Case Queries (Q1-Q16) on both DSMS and DBMS based solution

\begin{tabular}{|c|c|c|c|c|c|c|}
\hline \multirow{3}{*}{ Query \# } & \multicolumn{6}{|c|}{ Ease of Query Implementation } \\
\hline & \multicolumn{3}{|c|}{ DSMS } & \multicolumn{3}{|c|}{ DBMS } \\
\hline & Easy & Hard & Very Hard & Easy & Hard & Very Hard \\
\hline \multicolumn{7}{|l|}{ Evaluation } \\
\hline 1 & • & 0 & $\circ$ & • & o & $\circ$ \\
\hline 2 & - & $\circ$ & $\circ$ & • & o & $\circ$ \\
\hline 3 & • & o & o & o & o & • \\
\hline 7 & • & o & $\circ$ & $\circ$ & o & $\circ$ \\
\hline 8 & • & o & $\circ$ & $\circ$ & • & $\circ$ \\
\hline 9 & $\bullet$ & o & $\circ$ & • & o & $\circ$ \\
\hline \multicolumn{7}{|l|}{ Integration } \\
\hline 4 & • & 0 & $\circ$ & - & $\circ$ & $\circ$ \\
\hline 5 & • & $\circ$ & $\circ$ & • & $\circ$ & $\circ$ \\
\hline 6 & - & $\circ$ & $\circ$ & o & • & $\circ$ \\
\hline 10 & $\bullet$ & 0 & $\circ$ & • & 0 & $\circ$ \\
\hline 11 & - & $\circ$ & $\circ$ & $\circ$ & • & $\circ$ \\
\hline 12 & • & o & $\circ$ & 0 & $\bullet$ & $\circ$ \\
\hline 13 & $\bullet$ & 0 & $\circ$ & 0 & $\bullet$ & 0 \\
\hline 14 & $\bullet$ & 0 & 0 & $\bullet$ & 0 & 0 \\
\hline 15 & $\bullet$ & 0 & 0 & $\bullet$ & 0 & 0 \\
\hline 16 & $\bullet$ & 0 & 0 & 0 & 0 & $\bullet$ \\
\hline
\end{tabular}




\section{Evaluation}

\section{Query Language Evaluation}

Relative difficulty of implementing the Use-Case Queries (Q1-Q16) on both DSMS and DBMS based solution

\begin{tabular}{|c|c|c|c|c|c|c|}
\hline \multirow{3}{*}{ Query \# } & \multicolumn{6}{|c|}{ Ease of Query Implementation } \\
\hline & \multicolumn{3}{|c|}{ DSMS } & \multicolumn{3}{|c|}{ DBMS } \\
\hline & Easy & Hard & Very Hard & Easy & Hard & Very Hard \\
\hline \multicolumn{7}{|l|}{ Evaluation } \\
\hline 1 & $\bullet$ & ० & $\circ$ & $\bullet$ & ० & $\circ$ \\
\hline 2 & $\bullet$ & $\circ$ & $\circ$ & $\bullet$ & $\circ$ & $\circ$ \\
\hline 3 & $\bullet$ & $\circ$ & $\circ$ & o & $\circ$ & $\bullet$ \\
\hline 7 & $\bullet$ & ० & $\circ$ & o & ० & $\circ$ \\
\hline 8 & $\bullet$ & $\circ$ & $\circ$ & $\circ$ & $\bullet$ & $\circ$ \\
\hline 9 & $\bullet$ & $\circ$ & $\circ$ & $\bullet$ & $\circ$ & $\circ$ \\
\hline \multicolumn{7}{|c|}{ Integration } \\
\hline 4 & $\bullet$ & 0 & 0 & $\bullet$ & 0 & 0 \\
\hline 5 & • & 0 & 0 & $\bullet$ & 0 & 0 \\
\hline 6 & $\bullet$ & 0 & 0 & 0 & $\bullet$ & 0 \\
\hline 10 & $\bullet$ & 0 & $\circ$ & $\bullet$ & 0 & 0 \\
\hline 11 & • & 0 & 0 & 0 & • & 0 \\
\hline 12 & $\bullet$ & 0 & 0 & 0 & $\bullet$ & 0 \\
\hline 13 & $\bullet$ & 0 & $\circ$ & 0 & $\bullet$ & 0 \\
\hline 14 & $\bullet$ & 0 & 0 & $\bullet$ & 0 & 0 \\
\hline 15 & $\bullet$ & 0 & 0 & $\bullet$ & 0 & 0 \\
\hline 16 & $\bullet$ & 0 & 0 & 0 & 0 & $\bullet$ \\
\hline
\end{tabular}




\section{Evaluation}

\section{Query Language Evaluation}

Relative difficulty of implementing the Use-Case Queries (Q1-Q16) on both DSMS and DBMS based solution

\begin{tabular}{|c|c|c|c|c|c|c|}
\hline \multirow{3}{*}{ Query \# } & \multicolumn{6}{|c|}{ Ease of Query Implementation } \\
\hline & \multicolumn{3}{|c|}{ DSMS } & \multicolumn{3}{|c|}{ DBMS } \\
\hline & Easy & Hard & Very Hard & Easy & Hard & Very Hard \\
\hline \multicolumn{7}{|l|}{ Evaluation } \\
\hline 1 & $\bullet$ & 0 & ० & $\bullet$ & ० & ० \\
\hline 2 & $\bullet$ & $\circ$ & $\circ$ & $\bullet$ & $\circ$ & $\circ$ \\
\hline 3 & $\bullet$ & ० & ० & ० & ० & $\bullet$ \\
\hline 7 & $\bullet$ & ० & ○ & o & o & $\circ$ \\
\hline 8 & $\bullet$ & $\circ$ & $\circ$ & $\circ$ & $\bullet$ & $\circ$ \\
\hline 9 & $\bullet$ & $\circ$ & $\circ$ & $\bullet$ & $\circ$ & $\circ$ \\
\hline \multicolumn{7}{|l|}{ Integration } \\
\hline 4 & $\bullet$ & 0 & 0 & $\bullet$ & 0 & 0 \\
\hline 5 & $\bullet$ & 0 & 0 & • & 0 & 0 \\
\hline 6 & $\bullet$ & 0 & 0 & 0 & $\bullet$ & 0 \\
\hline 10 & $\bullet$ & 0 & 0 & • & 0 & 0 \\
\hline 11 & $\bullet$ & $\circ$ & $\circ$ & 0 & $\bullet$ & $\circ$ \\
\hline 12 & $\bullet$ & 0 & 0 & 0 & $\bullet$ & 0 \\
\hline 13 & $\bullet$ & 0 & 0 & 0 & • & 0 \\
\hline 14 & $\bullet$ & 0 & 0 & $\bullet$ & 0 & 0 \\
\hline 15 & $\bullet$ & 0 & 0 & • & 0 & 0 \\
\hline 16 & $\bullet$ & 0 & 0 & 0 & 0 & • \\
\hline
\end{tabular}




\section{Evaluation}

\section{Query Language Evaluation}

\section{Relative difficulty of implementing the Use-Case Queries (Q1-Q16) on both DSMS and DBMS based solution}

\begin{tabular}{|c|c|c|c|c|c|c|c|c|}
\hline \multirow{3}{*}{ Query\# } & \multicolumn{6}{|c|}{ Ease of Query Implementation } & \multicolumn{2}{|c|}{ Features Hindering Query Implementation in the DBMS } \\
\hline & \multicolumn{3}{|c|}{ DSMS } & \multicolumn{3}{|c|}{ DBMS } & \multirow{2}{*}{ Windowing Query } & \multirow{2}{*}{$\begin{array}{l}\text { Grouped/Agg. Query Projecting } \\
\text { Not Grouped/Agg. Attributes }\end{array}$} \\
\hline & Easy & Hard & Very Hard & Easy & Hard & Very Hard & & \\
\hline \multicolumn{9}{|l|}{ Evaluation } \\
\hline 1 & $\bullet$ & $\circ$ & $\circ$ & $\bullet$ & o & $\circ$ & o & o \\
\hline 2 & • & ○ & ○ & • & $\circ$ & $\circ$ & $\circ$ & $\circ$ \\
\hline 3 & $\bullet$ & o & $\circ$ & o & o & • & • & $\bullet$ \\
\hline 7 & $\bullet$ & o & $\circ$ & $\circ$ & $\circ$ & $\circ$ & o & $\circ$ \\
\hline 8 & $\bullet$ & o & $\circ$ & $\circ$ & $\bullet$ & $\circ$ & $\bullet$ & $\circ$ \\
\hline 9 & $\bullet$ & o & $\circ$ & • & $\circ$ & $\circ$ & $\circ$ & ० \\
\hline \multicolumn{9}{|l|}{ Integration } \\
\hline 4 & $\bullet$ & $\circ$ & $\circ$ & $\bullet$ & o & $\circ$ & o & o \\
\hline 5 & - & ○ & $\circ$ & - & $\circ$ & $\circ$ & o & $\circ$ \\
\hline 6 & • & 。 & $\circ$ & $\circ$ & $\bullet$ & $\circ$ & - & $\circ$ \\
\hline 10 & • & ○ & $\circ$ & • & $\circ$ & $\circ$ & $\circ$ & $\circ$ \\
\hline 11 & - & ० & $\circ$ & $\circ$ & - & $\circ$ & - & $\circ$ \\
\hline 12 & • & o & $\circ$ & $\circ$ & - & $\circ$ & - & $\circ$ \\
\hline 13 & $\bullet$ & $\circ$ & $\circ$ & $\circ$ & • & $\circ$ & $\bullet$ & $\circ$ \\
\hline 14 & $\bullet$ & o & $\circ$ & • & $\circ$ & $\circ$ & o & $\circ$ \\
\hline 15 & $\bullet$ & 0 & $\circ$ & - & o & 0 & o & o \\
\hline 16 & - & $\circ$ & $\circ$ & $\circ$ & 0 & - & - & - \\
\hline
\end{tabular}


Issue 1. Implementing Windows.

- DSMS Solution $\rightarrow$ Built-in Window Ops. (Easy)

- DBMS Solution $\rightarrow$ through Self-Join

(Hard) 


\section{Evaluation}

\section{Query Language Evaluation}

\section{Issue 2. Grouped/Aggregated Query Projecting Not Grouped/Aggregated Attributes.}

Example: "For each meter, return its current measurement, TS, and the AVG of measurements received so far." 


\section{Evaluation}

\section{Query Language Evaluation}

\section{Issue 2. Grouped/Aggregated Query Projecting Not Grouped/Aggregated Attributes.}

Example: "For each meter, return its current measurement, TS, and the AVG of measurements received so far."

\section{Batch Evaluation Approach}

DBMS One-Time Queries

SELECT Device, TS, Measure, AVG (Measure)

FROM InputDataStreamTable

GROUP BY Device

\begin{tabular}{|c|c|c|c|c|}
\hline \multirow{8}{*}{ 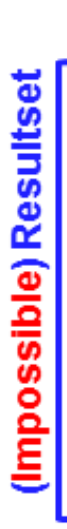 } & Device & TS & Measure & AVG(M.) \\
\hline & & $8: 10$ & 103 & \\
\hline & Lib. & $8: 11$ & 107 & 109 \\
\hline & & $8: 12$ & 117 & \\
\hline & & $8: 10$ & 23 & \\
\hline & A4 & $8: 11$ & 29 & 28 \\
\hline & & $8: 12$ & 32 & \\
\hline & lows & & & 2 Rows \\
\hline
\end{tabular}




\section{Evaluation}

\section{Query Language Evaluation}

\section{Issue 2. Grouped/Aggregated Query Projecting Not Grouped/Aggregated Attributes.}

Example: "For each meter, return its current measurement, TS, and the AVG of measurements received so far."

\section{Batch Evaluation Approach}

DBMS One-Time Queries

SELECT Device, TS, Measure, AVG(Measure) FROM InputDataStreamTable

GROUP BY Device

\begin{tabular}{|c|c|c|c|c|}
\hline \multirow{8}{*}{ 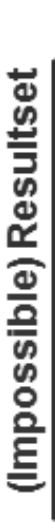 } & Device & TS & Measure & AVG(M.) \\
\hline & & $8: 10$ & 103 & \\
\hline & Lib. & $8: 11$ & 107 & 109 \\
\hline & & $8: 12$ & 117 & \\
\hline & & $8: 10$ & 23 & \\
\hline & A4 & 8:11 & 29 & 28 \\
\hline & & $8: 12$ & 32 & \\
\hline & Rows & & & 2 Rows \\
\hline
\end{tabular}

SELECT Device, TS, Measure, AVG(Measure) OVER W FROM InputDataStreamTable

WINDOW W AS (PARTITION BY Device ORDER BY TS ASC RANGE BETWEEN CURRENT ROW AND UNBOUNDED FOLLOWING)

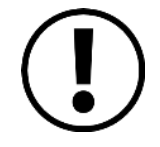
\begin{tabular}{|l|l|l|l|}
\hline Device & TS & Measure & AVG(M.) \\
\hline
\end{tabular}

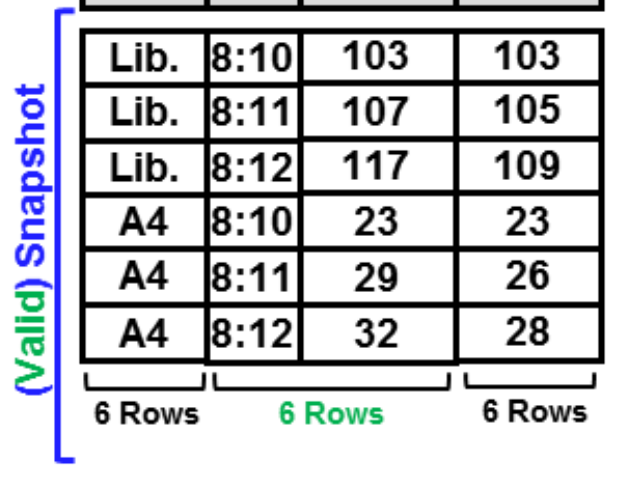




\section{Evaluation}

\section{Query Language Evaluation}

\section{Issue 2. Grouped/Aggregated Query Projecting Not Grouped/Aggregated Attributes.}

Example: "For each meter, return its current measurement, TS, and the AVG of measurements received so far."

\section{Incremental Evaluation Approach} DSMS Continuos Queries
SELECT Device, TS, Measure, AVG(Measure) FROM InputDataStream

GROUP BY Device

\begin{tabular}{l} 
Input Data Stream \\
\begin{tabular}{|c|c|c|c|c|c|c|}
\hline Device & $\mathrm{TS}$ & Measure \\
\hline Lib. & $8: 10$ & 103 \\
\hline A4 & $8: 10$ & 23 \\
\hline Lib. & $8: 11$ & 107 \\
\hline A4 & $8: 11$ & 29 \\
\hline Lib. & $8: 12$ & 117 \\
\hline A4 & $8: 12$ & 32 \\
\hline
\end{tabular} \\
\hline
\end{tabular}




\section{Evaluation}

\section{Query Language Evaluation}

\section{Practical Consequences of Issue 1 and Issue 2}

Use-Case Query Q16: For each meter, give me its current and expected energy consumption. Being the expected value of each meter given by the AVG consumption of the current hour, computed along last month.

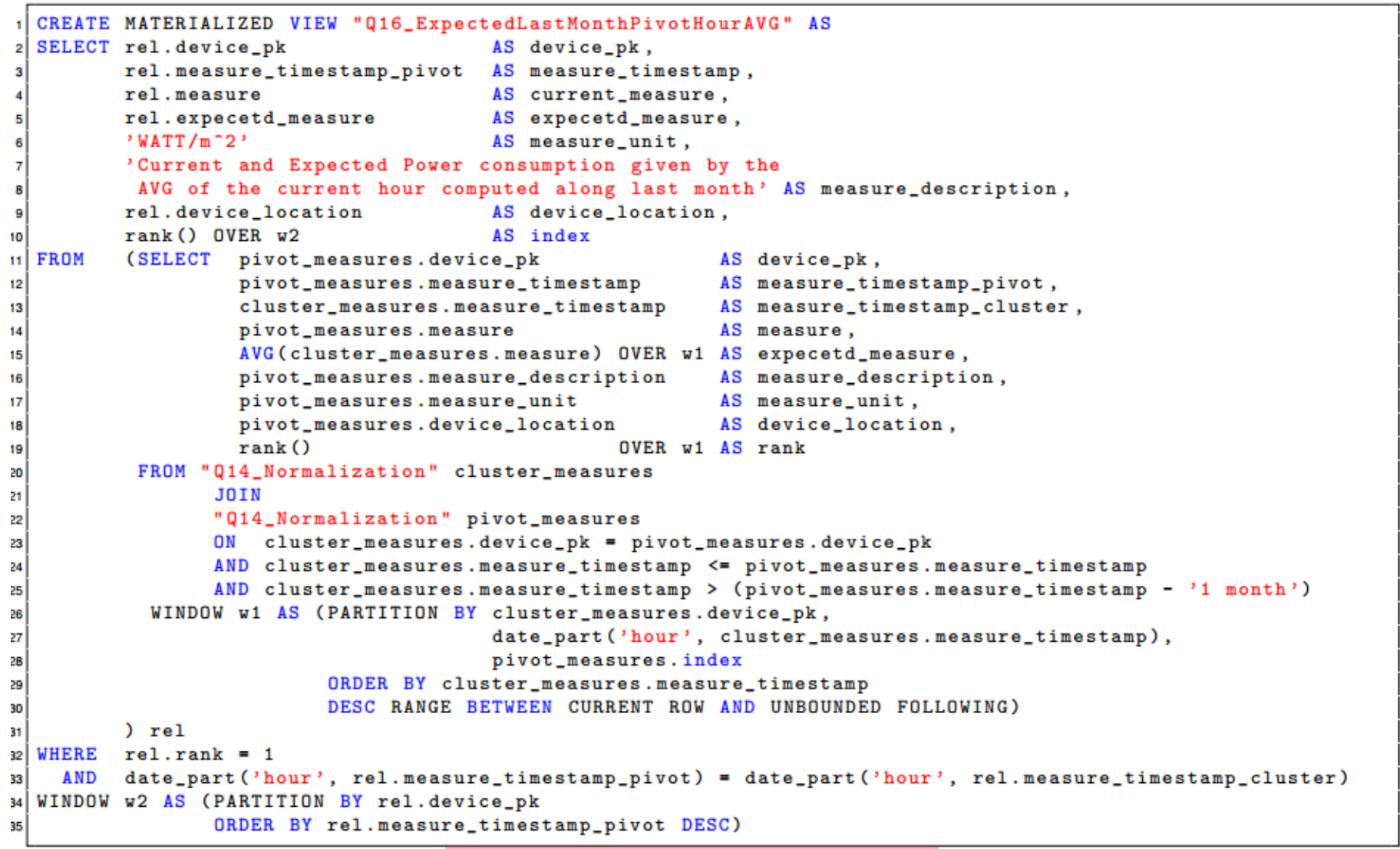

Q16 implementation in SQL (PostrgreSQL) 


\section{Evaluation}

\section{Query Language Evaluation}

\section{Practical Consequences of Issue 1 and Issue 2}

Use-Case Query Q16: For each meter, give me its current and expected energy consumption. Being the expected value of each meter given by the AVG consumption of the current hour, computed along last month.

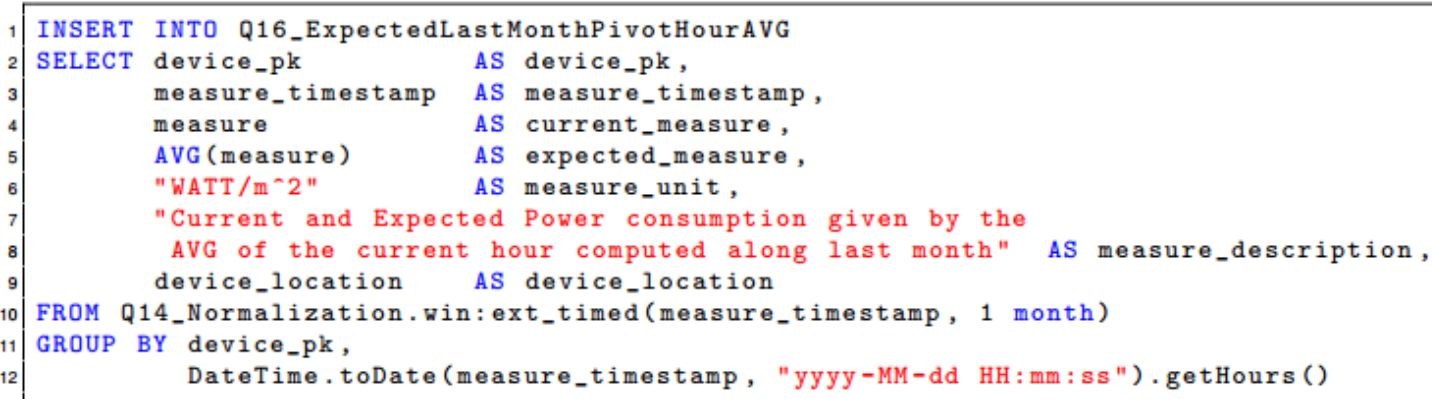

Q16 implementation in EPL (Esper) 


\section{Evaluation}

\section{Query Language Evaluation}

\section{Query Language Evaluation Results:}

DSMS's Query Language is most suitable to write and evaluate queries than SQL Query Language of DBMSS 


\section{Evaluation}

\section{Performance Evaluation}

Assess the ability of each solution to process the arriving energy metering data streams in real-time.

\section{Methodology:}

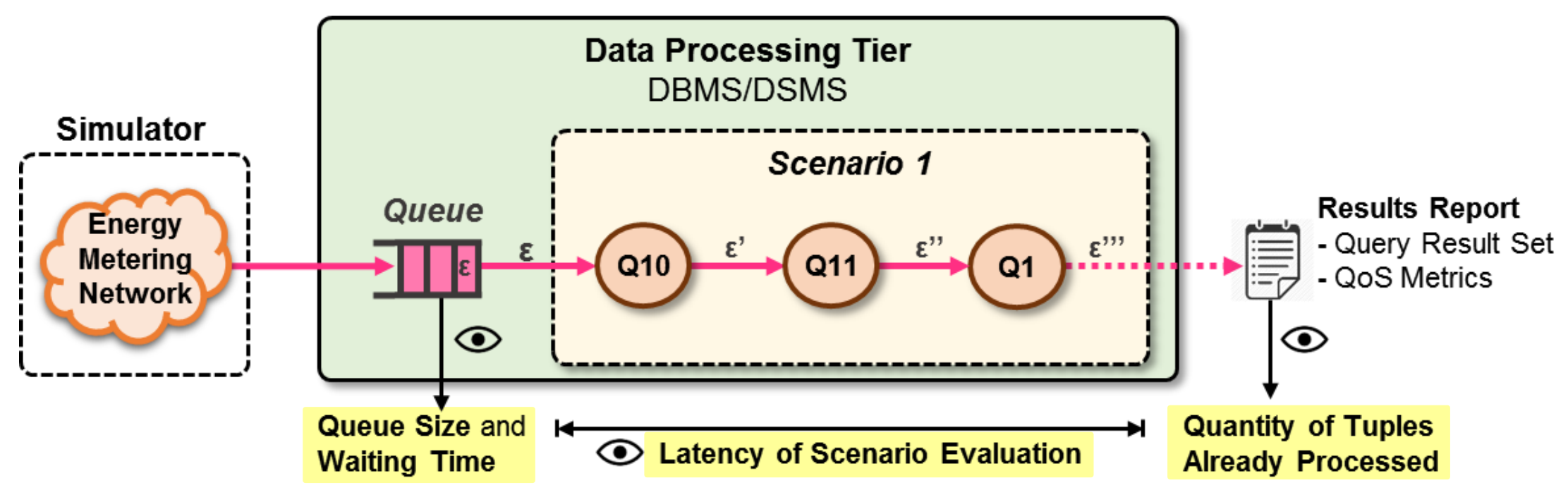




\section{Evaluation}

\section{Performance Evaluation}

Assess the ability of each solution to process the arriving energy metering data streams in real-time.

\section{Methodology:}
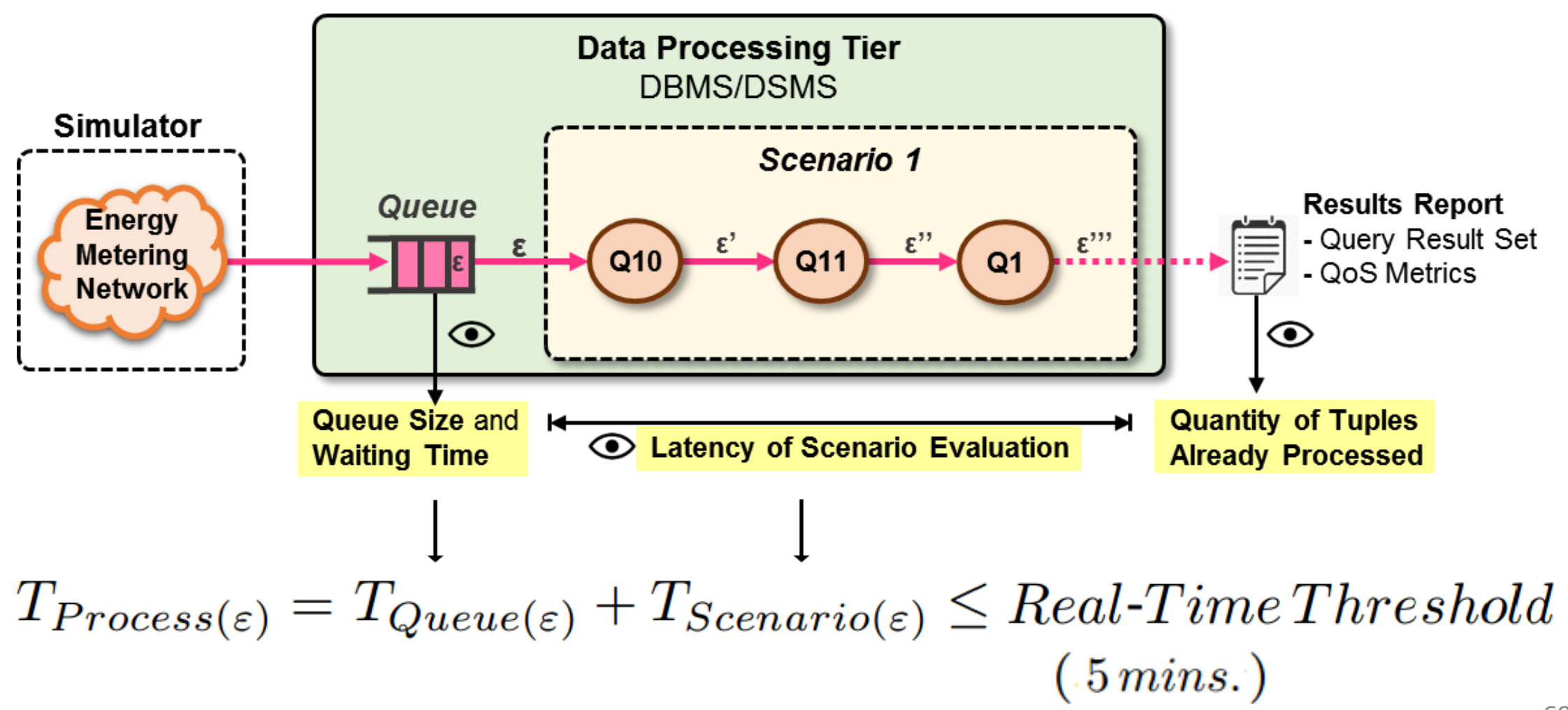


\section{Evaluation}

\section{Performance Evaluation}

\section{Experimental Setup:}

- 9 Scenarios Evaluated (one at a time)

- 18 Tests as a Total (DBMS Sol. + DSMS Sol.) 


\section{Evaluation}

\section{Performance Evaluation}

\section{Experimental Setup:}

- 9 Scenarios Evaluated (one at a time)

- 18 Tests as a Total (DBMS Sol. + DSMS Sol.)

- 10-hour duration for each test

- 8 energy meters

- Each meter: 4 measurements/minute

- Total of 19200 measurements delivered along 10 hours

(57600 tuples, 3-Phase current) 


\section{Evaluation}

\section{Performance Evaluation}

\section{Experimental Setup:}

- 9 Scenarios Evaluated (one at a time)

- 18 Tests as a Total (DBMS Sol. + DSMS Sol.)

- 10-hour duration for each test

- 8 energy meters

- Each meter: 4 measurements/minute

- Total of 19200 measurements delivered along 10 hours

(57600 tuples, 3-Phase current)

- 1,875 secs., average arrival period of each measurement 


\section{Evaluation}

\section{Performance Evaluation - Scalability of Scenario Evaluation Latency}

Results of the Experiments: $T_{\text {Process }(\varepsilon)}=T_{\text {Queue }(\varepsilon)}+T_{\text {Scenario }(\varepsilon)}$

Scenario 3: "Identify the energy meters reporting an energy consumption (Motivation Q1) 20\% above the respective average over the last 24 hours."

Performance Evaluation

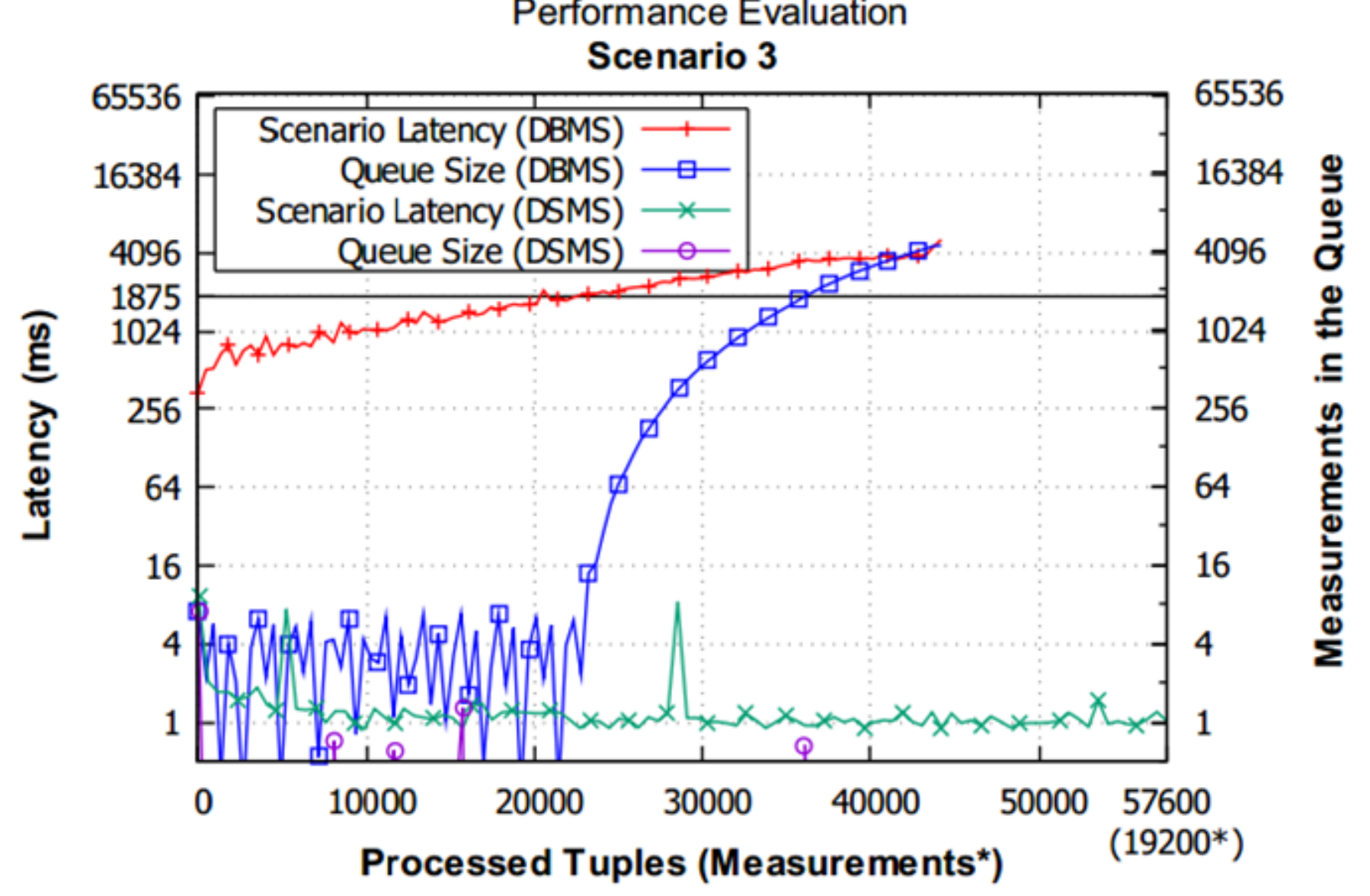




\section{Evaluation}

\section{Performance Evaluation - Scalability of Scenario Evaluation Latency}

Results of the Experiments: $T_{\text {Process }(\varepsilon)}=T_{\text {Queue }(\varepsilon)}+T_{\text {Scenario }(\varepsilon)}$

Scenario 3: "Identify the energy meters reporting an energy consumption (Motivation Q1) 20\% above the respective average over the last 24 hours."

Performance Evaluation

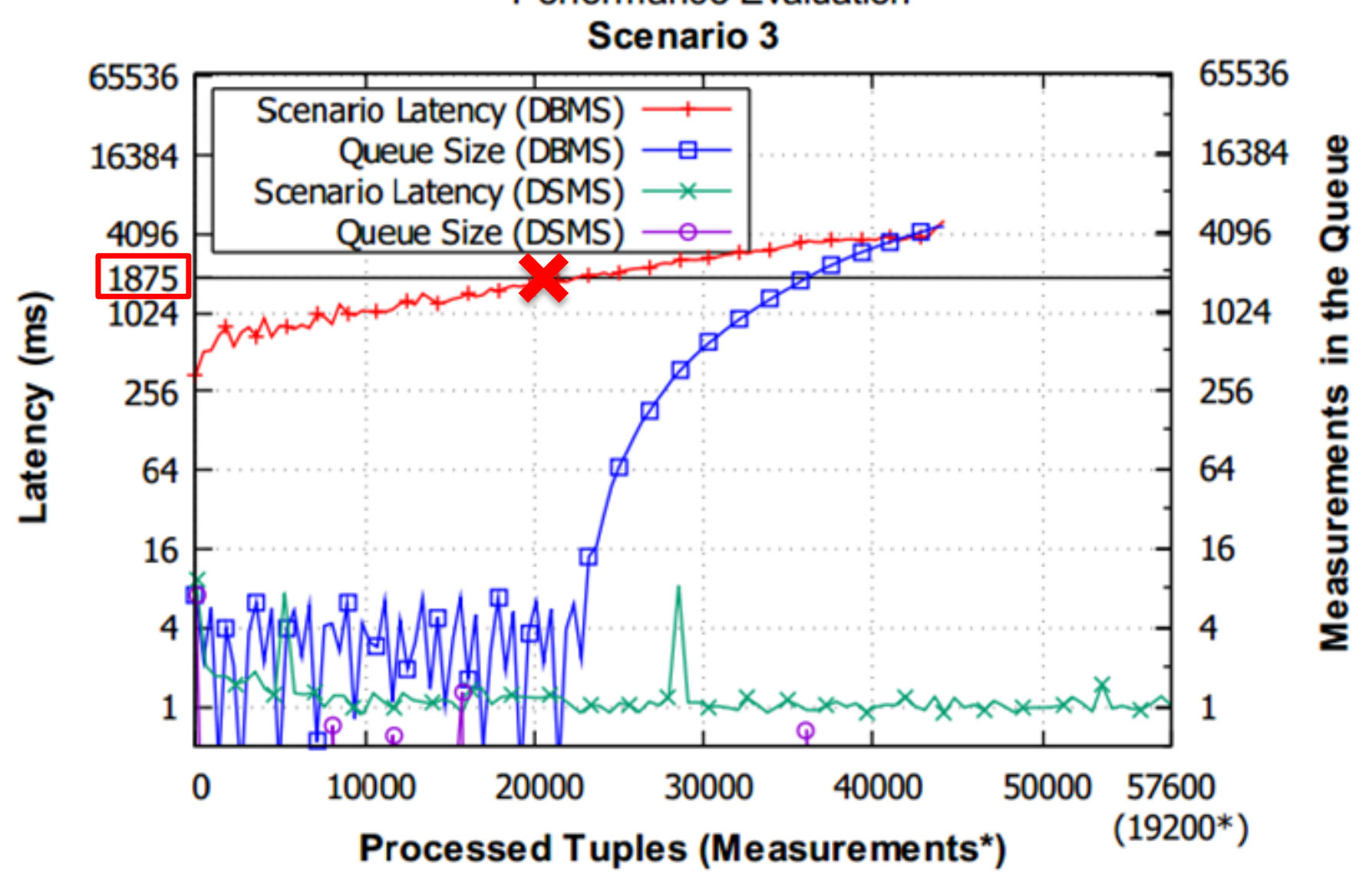




\section{Evaluation}

\section{Performance Evaluation - Scalability of Scenario Evaluation Latency}

Results of the Experiments: $T_{\text {Process }(\varepsilon)}=T_{\text {Queue }(\varepsilon)}+T_{\text {Scenario }(\varepsilon)}$

Scenario 3: "Identify the energy meters reporting an energy consumption (Motivation Q1) 20\% above the respective average over the last 24 hours."

Performance Evaluation

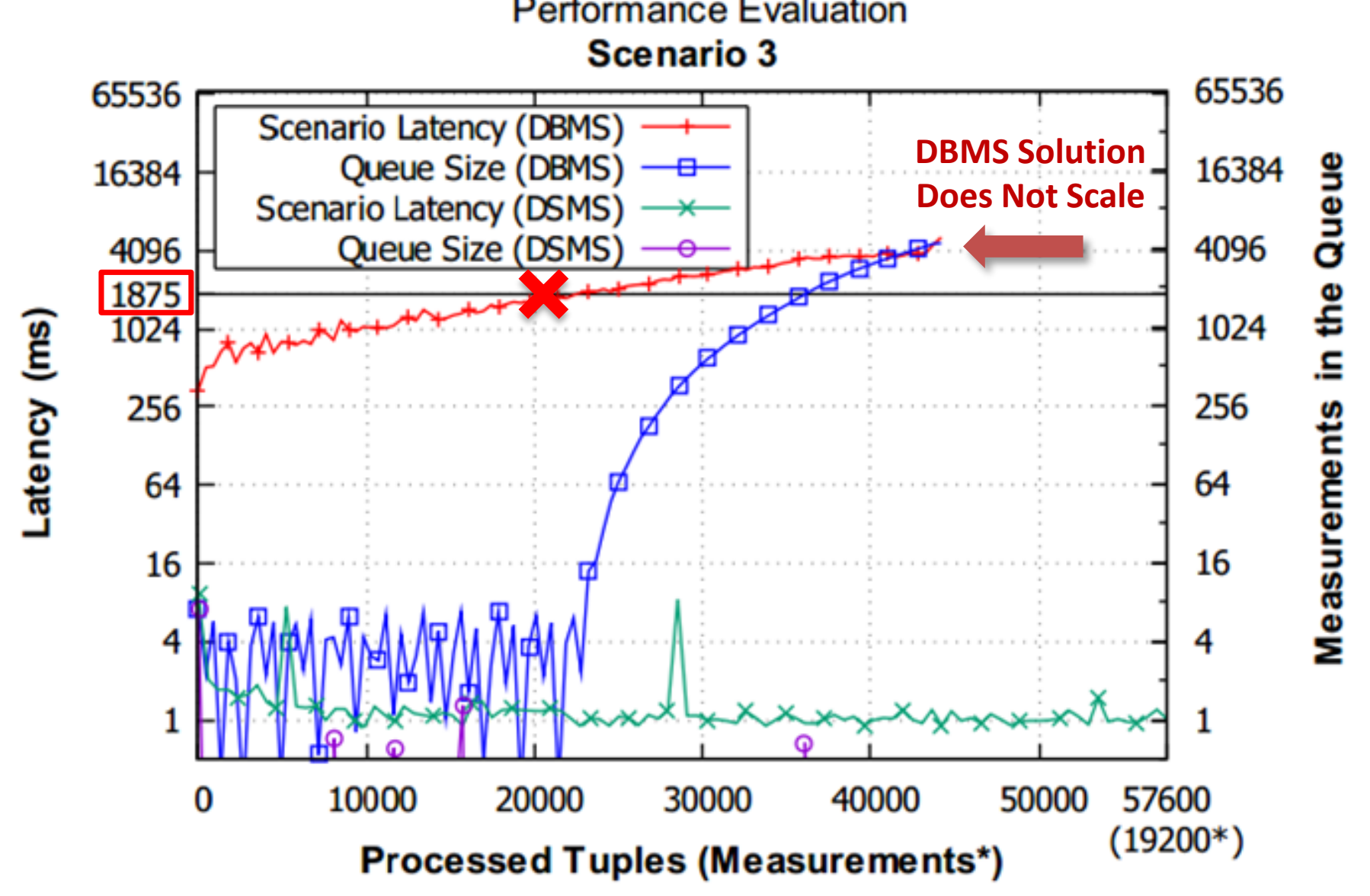




\section{Evaluation}

\section{Performance Evaluation - Scalability of Scenario Evaluation Latency}

Results of the Experiments: $T_{\text {Process }(\varepsilon)}=T_{\text {Queue }(\varepsilon)}+T_{\text {Scenario }(\varepsilon)}$

Scenario 3: "Identify the energy meters reporting an energy consumption (Motivation Q1) 20\% above the respective average over the last 24 hours."

Performance Evaluation

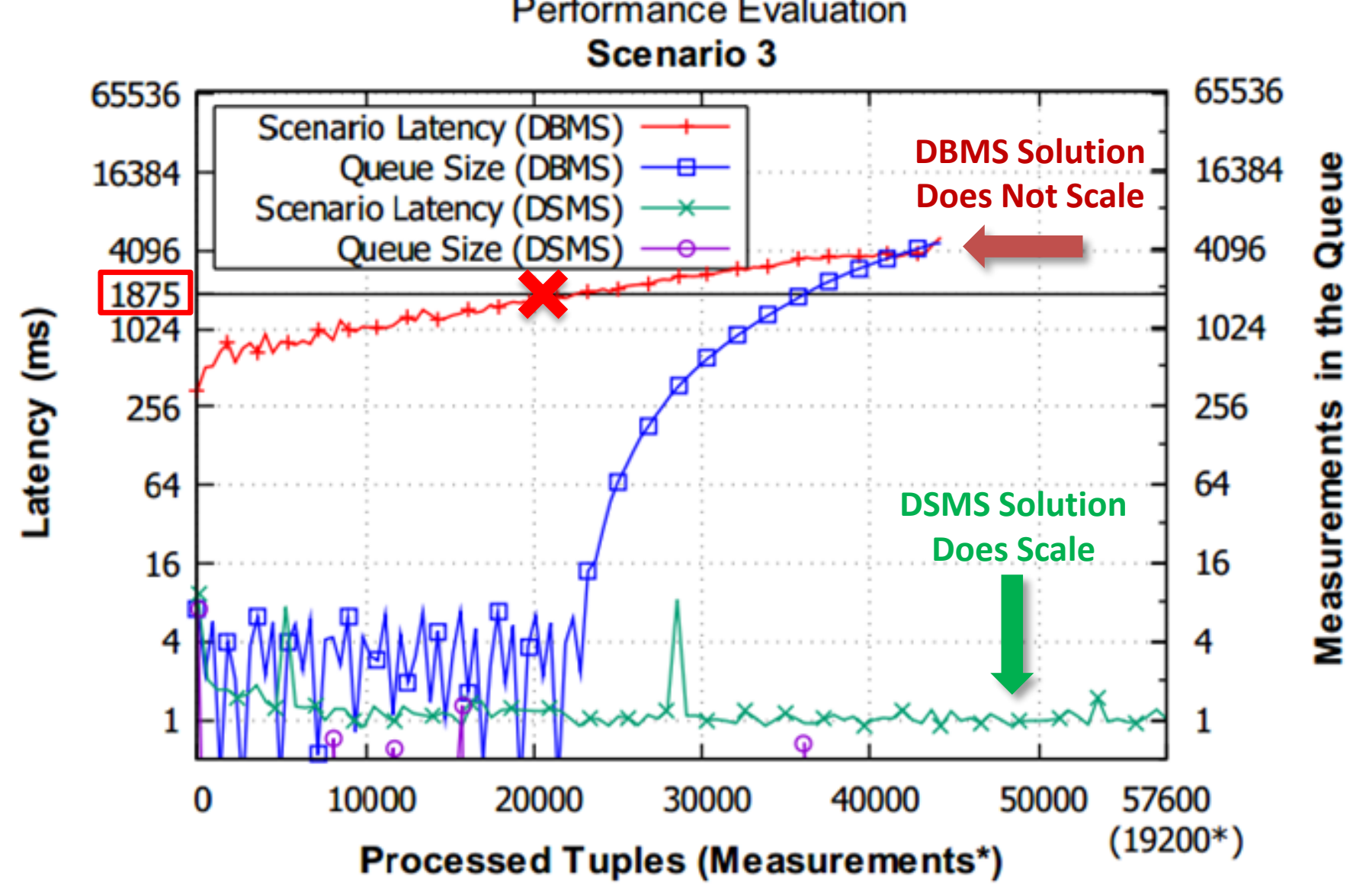




\section{Evaluation}

\section{Performance Evaluation - Evolution of Queue Waiting Time}

\section{Results of the Experiments: $T_{\text {Process }(\varepsilon)}=T_{\text {Queue }(\varepsilon)}+T_{\text {Scenario }(\varepsilon)}$}

Scenario 3: "Identify the energy meters reporting an energy consumption (Motivation Q1) 20\% above the respective average over the last 24 hours."

(a) Time a measurement had to wait in the queue to be processed DBMS Solution

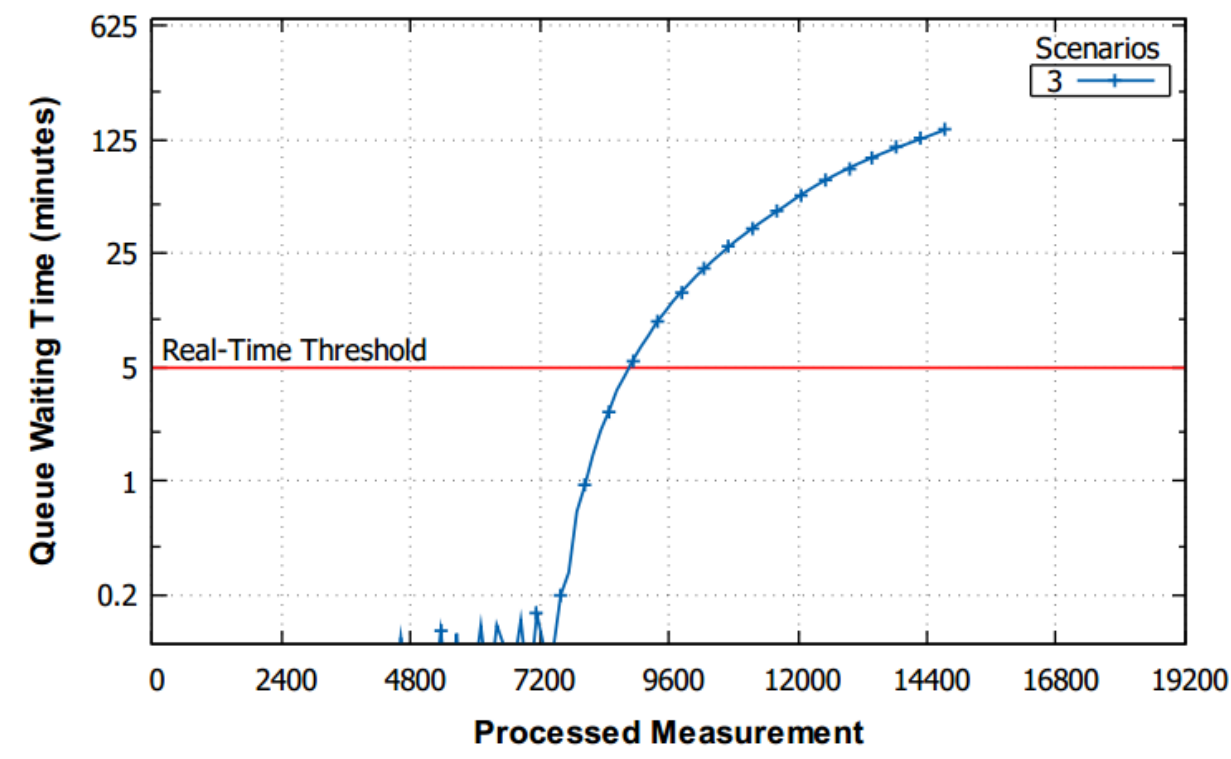

(b) Time a measurement had to wait in the queue to be processed DSMS Solution

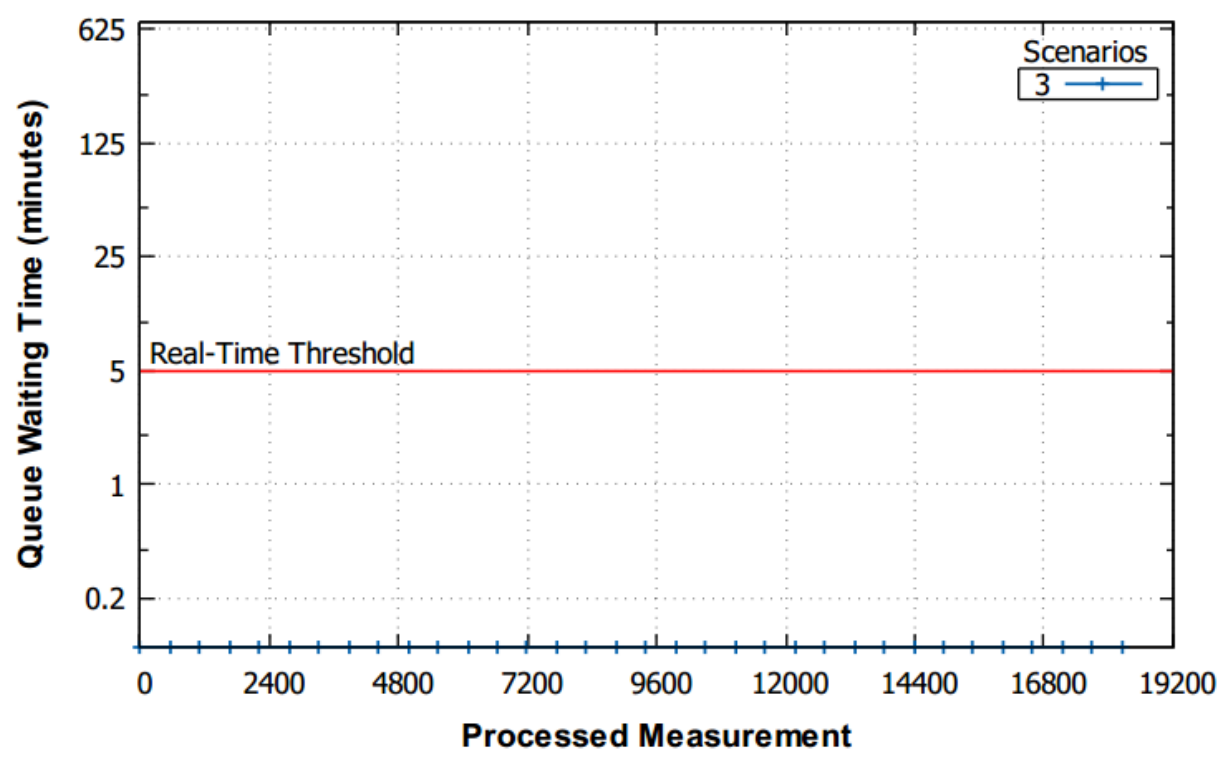




\section{Evaluation}

\section{Performance Evaluation - Scalability of Evaluation Latency for all 9 Scenarios}

\section{Results of the Experiments: $T_{\operatorname{Process}(\varepsilon)}=T_{\text {Queue }(\varepsilon)}+T_{\text {Scenario }(\varepsilon)}$}
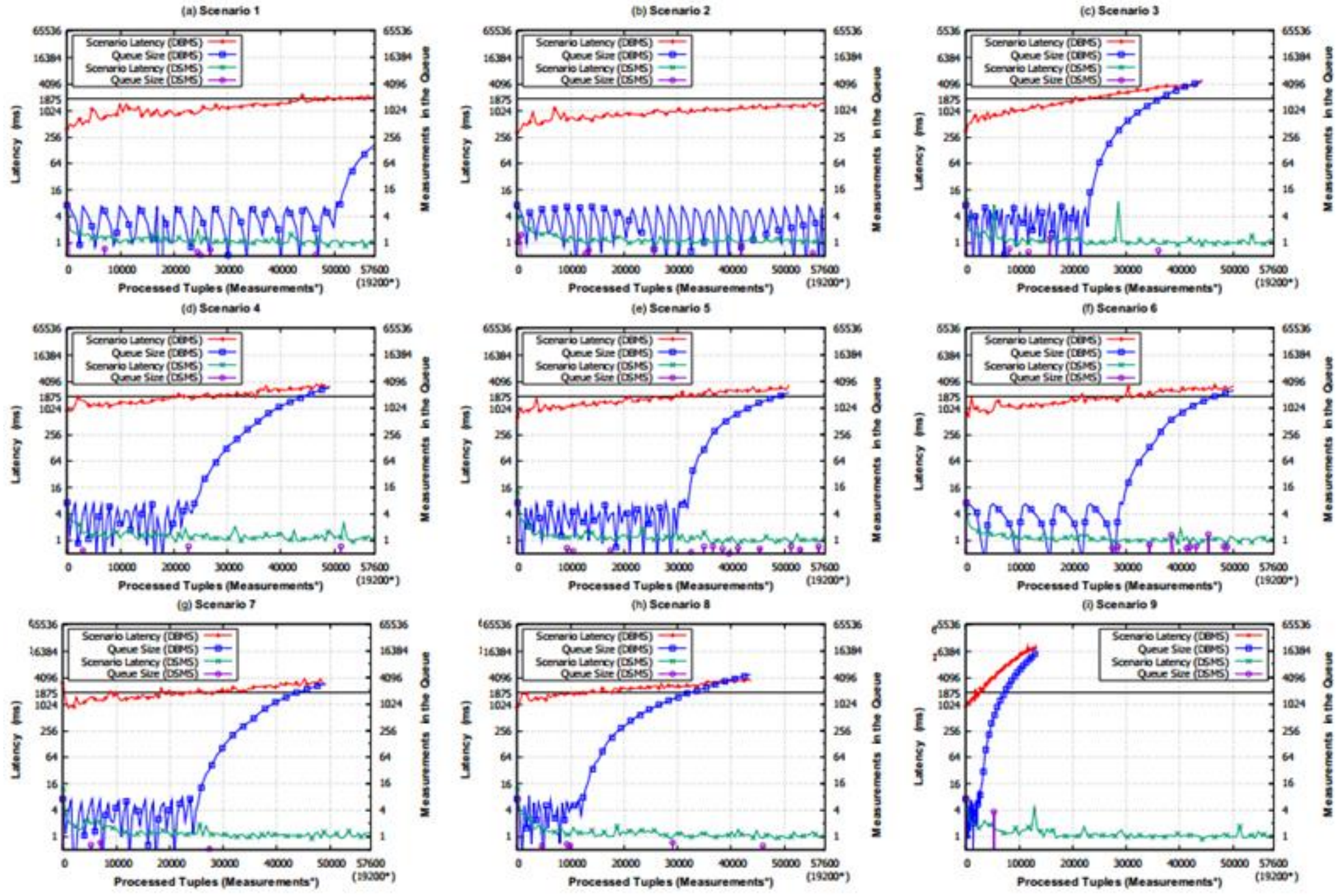


\section{Evaluation}

\section{Performance Evaluation - Evolution of Queue Waiting Time for all 9 Scenarios}

\section{Results of the Experiments: $T_{\text {Process }(\varepsilon)}=T_{\text {Queue }(\varepsilon)}+T_{\text {Scenario }(\varepsilon)}$}

Scenario 3: "Identify the energy meters reporting an energy consumption (Motivation Q1) 20\% above the respective average over the last 24 hours."

(a) Time a measurement had to wait in the queue to be processed DBMS Solution

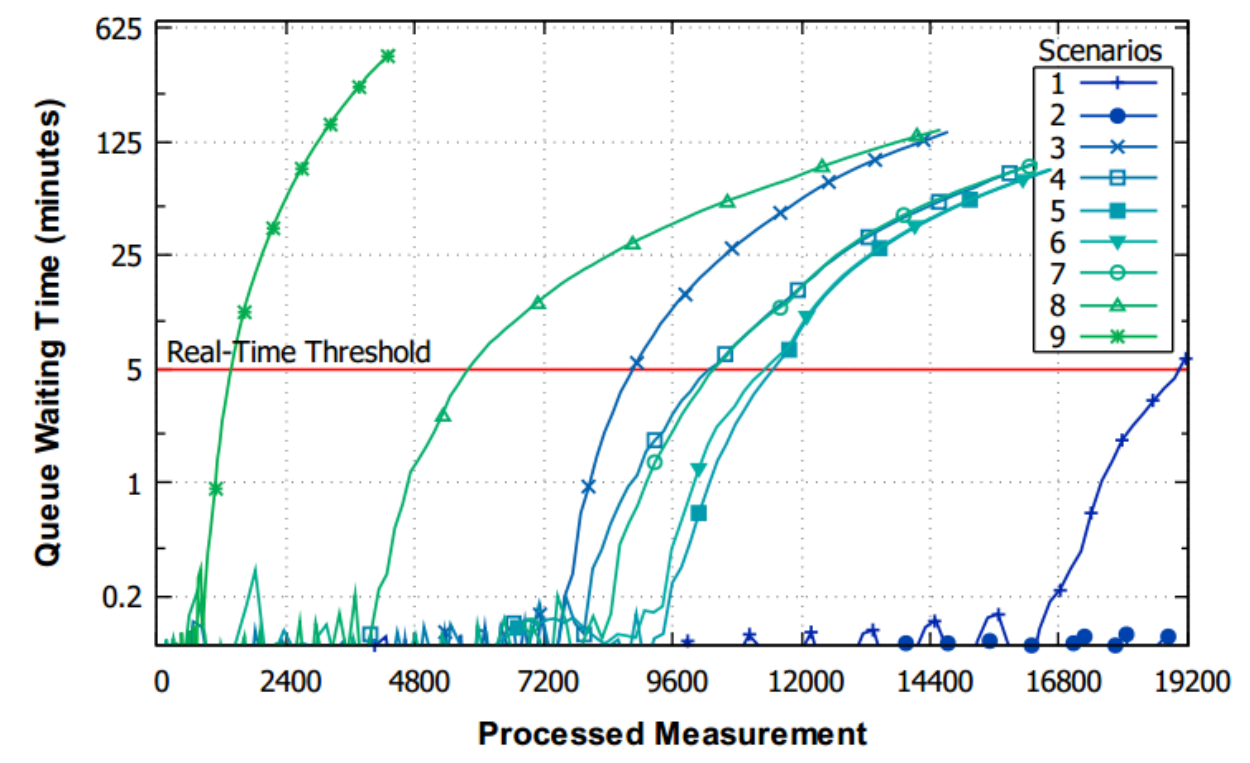

(b) Time a measurement had to wait in the queue to be processed DSMS Solution

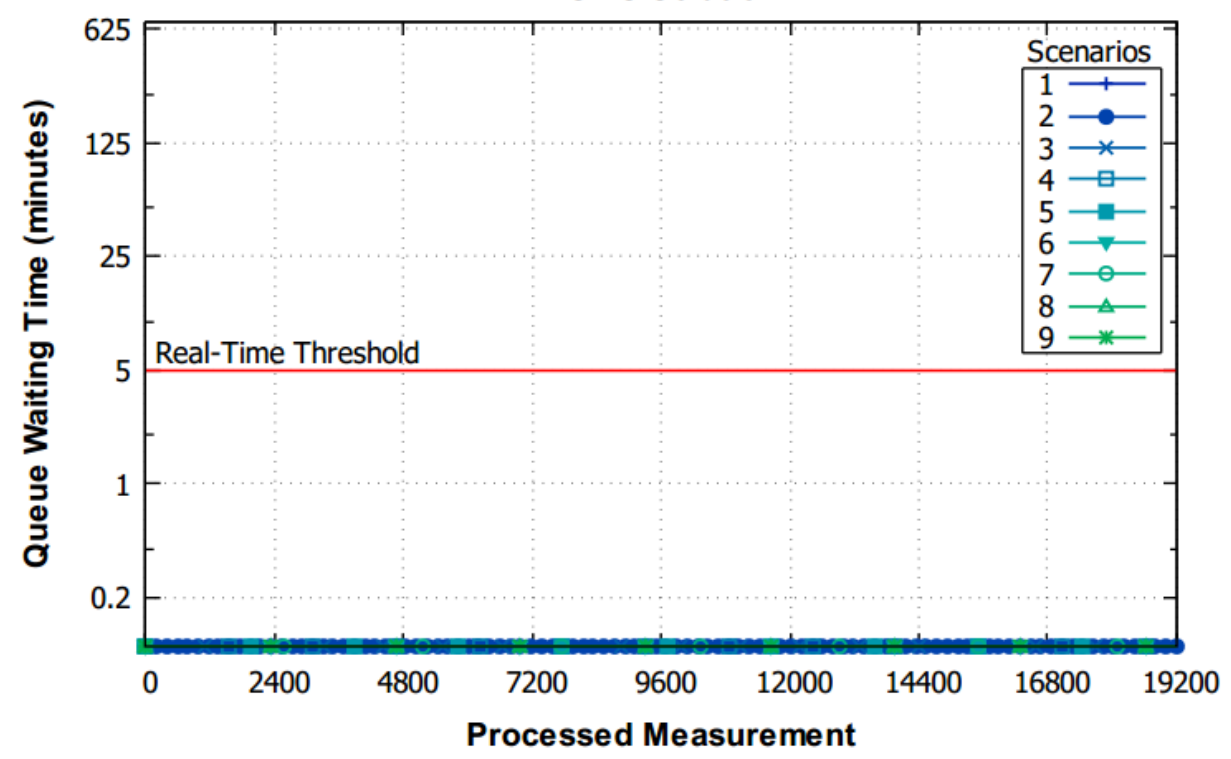




\section{Evaluation}

\section{Performance Evaluation}

Performance Evaluation Results:

- DBMS Solution failed to timely evaluate energy metering data in $\mathbf{8}$ of $\mathbf{9}$ Scenarios

- DSMS Solution successfully evaluated in RT the energy metering data in all 9 Scenarios 


\section{Evaluation}

\section{Performance Evaluation}

Performance Evaluation Results:

- DBMS Solution failed to timely evaluate energy metering data in $\mathbf{8}$ of $\mathbf{9}$ Scenarios

- DSMS Solution successfully evaluated in RT the energy metering data in all 9 Scenarios

An EMS aiming at processing energy metering data in real-time must be supported by a DSMS, instead of by a DBMS 


\section{Agenda}

- Introduction

- State of the art

- Motivation

- Problem Statement

- Proposed Solution

- Evaluation

- Conclusion

- Bibliography 


\section{Conclusion}

- Current EMSs are using a DBMSs to process sensor data, preventing them to monitor energy metering networks in real-time.

- DSMSs are used to process data streams efficiently in several domains.

- We introduced an EMS's Data Processing Architecture supported by a DSMS.

- Benchmark evaluation show that our solution outperformed the state of the art approach in both data evaluation latency and query language expressibility

- We validated our hypothesis that, to process energy metering data streams in real-time, EMSs should be supported by DSMSs, instead of DBMSs. 


\section{Conclusion}

\section{Published Article - IEEE International Congress on Big Data}

IEEE.org | IEEE Xplore Digital Library | IEEE-SA | IEEE Spectrum | More Sites Cart (0) | Create Account | Personal Sign In

\section{IEEE Xplore}

Digital Library

\section{- IEEE}

\begin{tabular}{|l|l|l|l|}
\hline BROWSE $\checkmark$ & MY SETtings $\checkmark$ & GET HELP $\checkmark$ & WHAT CAN I ACCESS? \\
\hline Enter Search Term & \\
\hline Basic Search & Author Search & Publication Search & \multirow{2}{*}{ Advanced Search $\mid$ Other Search Options $\checkmark$} \\
\hline
\end{tabular}

Browse Conference Publications > Big Data (BigData Congress), ...

\section{Real-Time Integration of Building} Energy Data

\section{눤 Full Text as PDF}

[0 Full Text in HTML

3 Author(s)

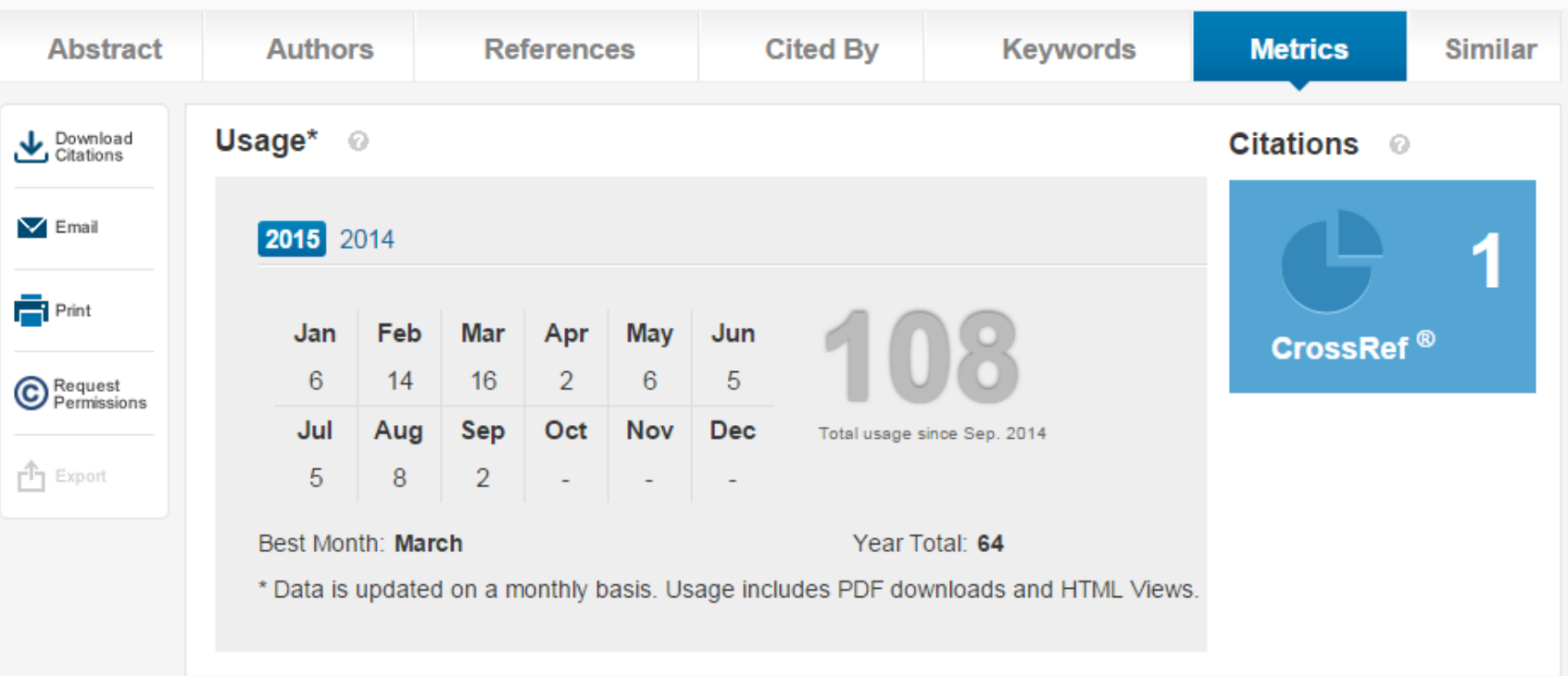




\section{Conclusion}

\section{Published Article - IEEE International Congress on Big Data}

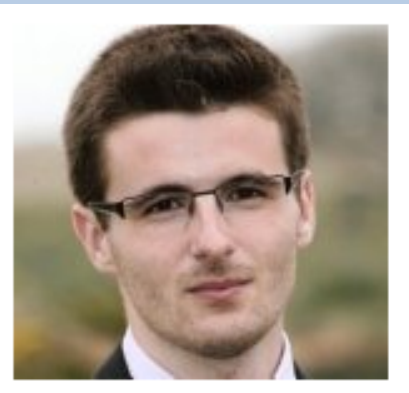

\section{Diogo Anjos}

Instituto Superior Técnico, INESC-ID Lisboa

Software Engineering, Data Engineering, Data Stream Processing

E-mail confirmado em tecnico.ulisboa.pt

O meu perfil é público

\begin{tabular}{llcc}
\hline Título & T Adicionar & Citado por & Ano \\
\hline $\begin{array}{l}\text { Real-Time Integration of Building Energy Data } \\
\text { D Anjos, P Carreira, AP Francisco }\end{array}$ & 3 & 2014 \\
2014 IEEE International Congress on Big Data (BigData Congress) & & \\
\hline
\end{tabular}

\section{Google Académico}

Índices de citações Todos Desde 2010

Citações 3

Índice $\mathrm{h}$

Índice i10

1

3

Indice i10

0

2015

\section{Coautores}

Paulo Carreira

A P Francisco 


\section{Conclusion}

\section{Published Article - IEEE International Congress on Big Data}

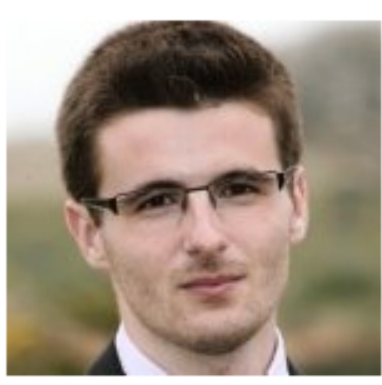

\section{Diogo Anjos}

Instituto Superior Técnico, INESC-ID Lisboa

Software Engineering, Data Engineering, Data Stream Processing

E-mail confirmado em tecnico.ulisboa.pt

O meu perfil é público

$\checkmark$ Seguir $>$ Google Académico

Índices de citaçöes Todos Desde 2010

Citações 3

Índice $\mathrm{h}$

Índice $\mathrm{i} 10$

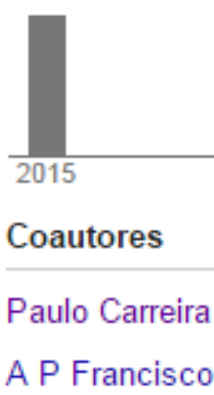

\section{Real-Time Integration of Building Energy Data}

$\square$ D Anjos, P Carreira, AP Francisco

2014 IEEE International Congress on Big Data (BigData Congress)
Citado por Ano

32014

home | browse | search | about

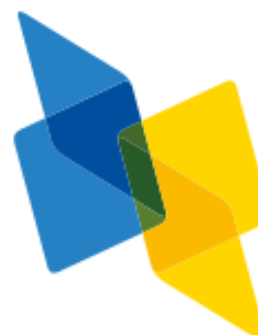

dblp

computer science bibliography
- 2 search dblp

\section{${ }_{[-]}^{[+]}$Diogo Anjos $\perp$ \&}

$>$ Home $>$ Persons

- by year $\cong \sim$ Trier 1

[-] 2010 - today 0

$[+]$ Refine list

2014

- [c1] 国 斗 穴 Diogo Anjos, Paulo Carreira, Alexandre P. Francisco:

Real-Time Integration of Building Energy Data. BigData Congress 2014: 250-257

$[-]$ Coauthor Index $\mathbb{1} \boldsymbol{\theta}$ 


\title{
Conclusion
}

\section{2nd Article Submitted to ICDE16}

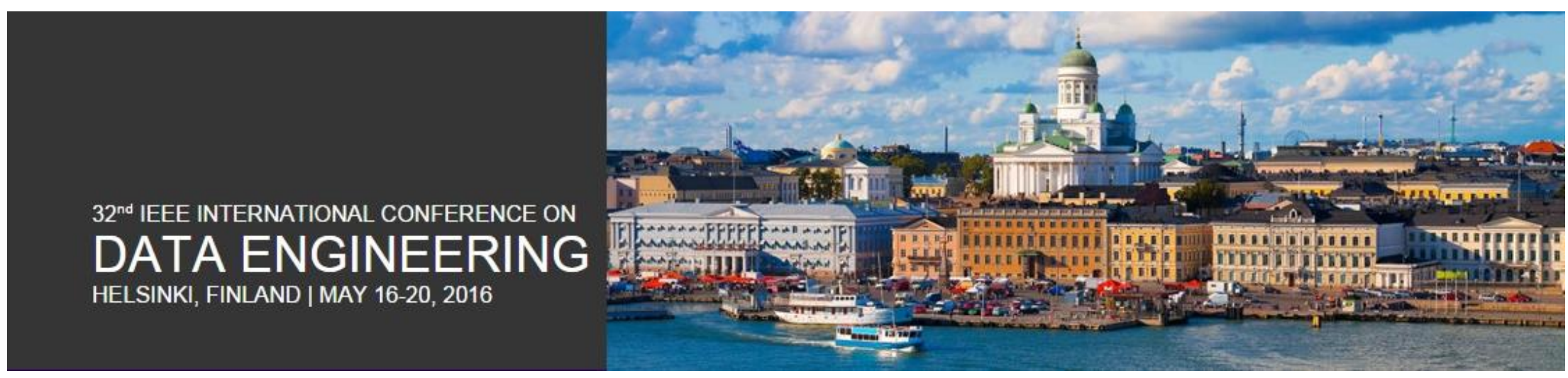

\section{Real-Time Monitoring of Building Energy Metering Networks}

\author{
Diogo Anjos \\ INESC-ID Lisboa \\ Instituto Superior Técnico (IST) \\ Universidade de Lisboa \\ Lisbon, Portugal \\ diogo.silva.anjos@tecnico.ulisboa.pt
}

\author{
Paulo Carreira \\ INESC-ID Lisboa \\ Instituto Superior Técnico (IST) \\ Universidade de Lisboa \\ Lisbon, Portugal \\ paulo.carreira@tecnico.ulisboa.pt
}

Alexandre P. Francisco INESC-ID Lisboa

Instituto Superior Técnico (IST)

Universidade de Lisboa

Lisbon, Portugal

aplf@tecnico.ulisboa.pt

\begin{abstract}
Energy Management Systems (EMSs) are used to monitor energy consumption in buildings with the purpose of improving energy efficiency, by identifying savings opportunities and misuse situations. To achieve that, an EMS collects energy metering data streams from a network of energy meters in the

required information to determine the adjustments towards improving energy usage [4].

One fundamental aspect of energy management is timeliness: faster decisions translate to less waste and larger savings.
\end{abstract}




\section{Real-time Integration of Building Energy Data}

diogo.silva.anjos@tecnico.ulisboa.pt

November 20, 2015 


\section{Bibliography (1 of 3)}

D.Anjos, P.Carreira, and A.P.Francisco. Real-time integration of building energy data. In Big Data (BigData Congress), 2014 IEEE International Congress on, pages 250-257. IEEE, 2014.

N.Akhtar and F.H.Siddiqui. UDP packet monitoring with stanford data stream manager. In 2011 International Conference on Recent Trends in Information Technology (ICRTIT), pages 533-537. leee, June 2011.

B.Babcock, S.Babu, M.Datar, R.Motwani, and J.Widom. Models and issues in data stream systems. In Proceedings of the twenty-first ACM SIGMOD-SIGACT-SIGART symposium on Principles of database systems, pages 1-16. ACM, 2002

C.Bizer and A.Schultz. The Berlin sparql benchmark. 2009.

P.Bonnet, J.Gehrke, and P.Seshadri. Towards sensor database systems. In Mobile Data Management, pages 3-14. Springer, 2001.

C.Cranor, T.Johnson, and O.Spataschek. Gigascope : A Stream Database for Network Applications. In Proceedings of the 2003 ACM SIGMOD International Conference on Management of Data, pages 647-651. ACM, 2003. ISBN 158113634X. 


\section{Bibliography (2 of 3)}

B.Chandramouli, M.Ali, J.Goldstein, B.Sezgin, and B.S.Raman. Data stream management systems for computational finance. Computer, (12):45-52, 2010

L.Golab and M.T.Özsu. Issues in data stream management. ACM Sigmod Record, 32(2):5-14, 2003.

J.Granderson, M.Piette, B.Rosenblum, and et al. L.Hu. Energy Information Handbook: Applications for Energy-Efficient Building Operations. Lawrence Berkeley National Laboratory, LBNL-5272E., 2011

X.Jiang, S.Yoo, and J.Choi. Dsms in ubiquitous-healthcare: A borealis-based heart rate variability monitor. In Biomedical Engineering and Informatics (BMEI), 2011 4th International Conference on, volume 4, pages 21442147. IEEE, 2011

A.H.Kazmi, M.J.O'grady, D.T.Delaney, A.G.Ruzzelli, and G.M.P.O'hare. A review of wirelesssensor-networkenabled building energy management systems. ACM Trans. Sen. Netw., 10(4):66:1-66:43, 2014.

X.Li, B.Plale, N.Vijayakumar, R.Ramachandran, S.Graves, and H.Conover. Real-time storm detection and weather forecast activation through data mining and events processing. Earth Science Informatics, 1(2):49-57, 2008 


\section{Bibliography (3 of 3)}

S.Madden and M.J.Franklin. Fjording the Stream : An Architecture for Queries over Streaming Sensor Data. In Data Engineering, 2002. Proceedings. 18th IEEE International Conference on, pages 555-566, 2002.

A.Mukherjee, P.Diwan, P.Bhattacharjee, D.Mukherjee, and P.Misra. Capital market surveillance using stream processing. In Computer Technology and Development (ICCTD), 2010 2nd International Conference on, pages 577-582. IEEE, 2010.

L.Pérez-Lombard, J.Ortiz, and C.Pout. A review on buildings energy consumption information. Energy and Buildings, 40(3):394-398, Jan. 2008.

Q.Zhang, C.Pang, S.Mcbride, D.Hansen, C.Cheung, and M.Steyn. Towards health data stream analytics. In Complex Medical Engineering (CME), 2010 IEEE/ICME International Conference on, pages 282-287. IEEE, 2010. 


\section{Backup Slides}




\section{Evaluation}

\section{Methodology}

Two ortogonal implementations of

Proposed Data Processing Architecture

Benchmark Evaluation

- All 9 Use-Case Scenarios were implemented in both DSMS and DBMS solutions

- To feed these scenarios with energy metering data streams we implement a Simulator of Taguspark Energy Metering Nw.

I. Deterministic Datasets

II. Configurable Time Ranges

III.Configurable Load

Yet, still a real-world dataset

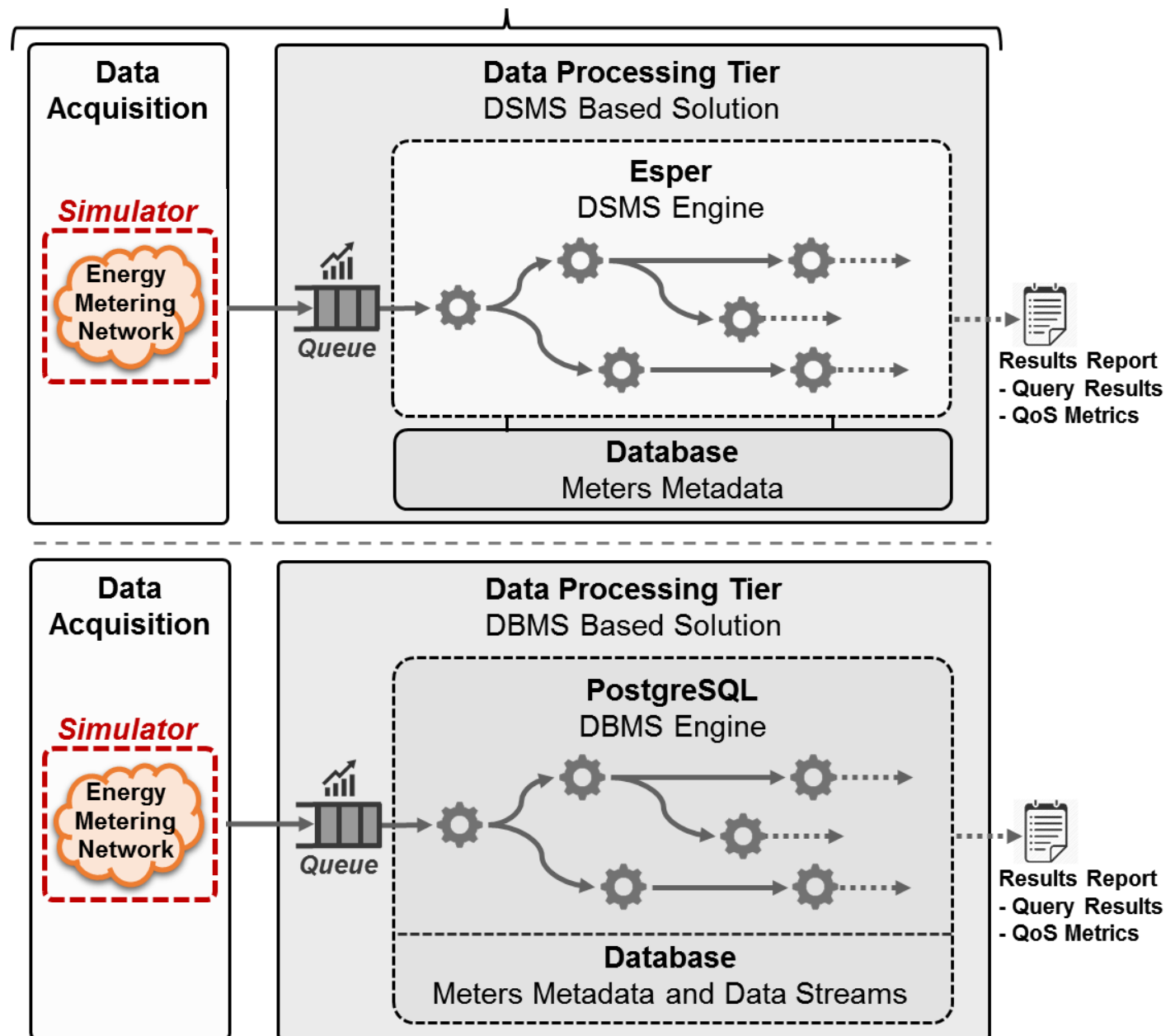

9 GitHub https://github.com/diogo-gsa/data-processing-architecture 


\section{Problem Statement}

- The motivation of this research work:

"EMSs must be capable of processing energy metering data in Real-Time, to improve energy efficiency in buildings."

- The problem identified by this research work:

"By being supported by DBMSs, current EMSs are struggling to process energy metering data streams in real-time."

- This dissertation aims to validate the thesis that: "An EMS supported by a DSMS outperforms an EMS supported by a DBMS, on processing energy metering data in real-time."

Better

Performance i. Lower Query Evaluation Latency

ii. Greater Query Language Expressibility 


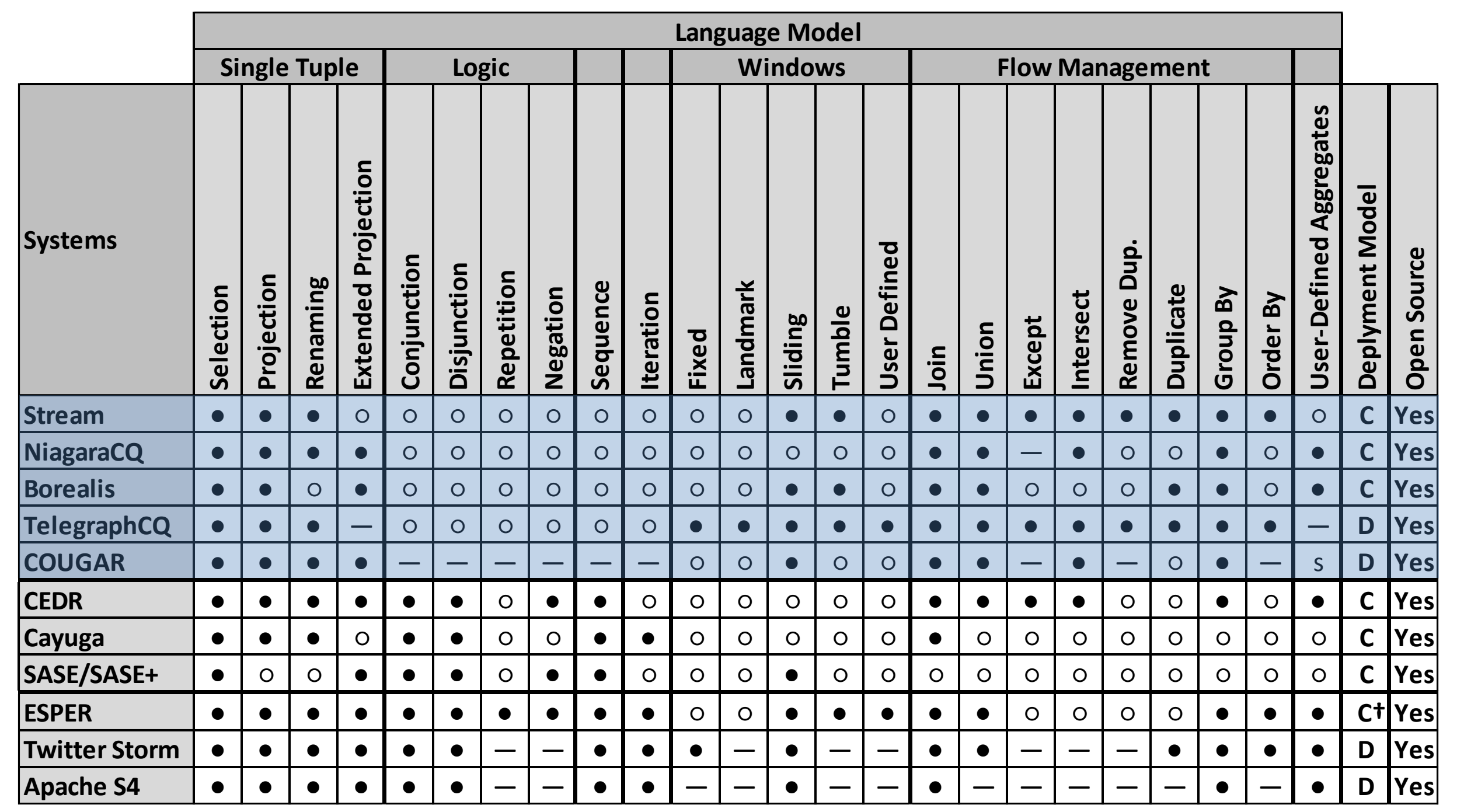




\section{Related Work}

Data Stream

Event Stream

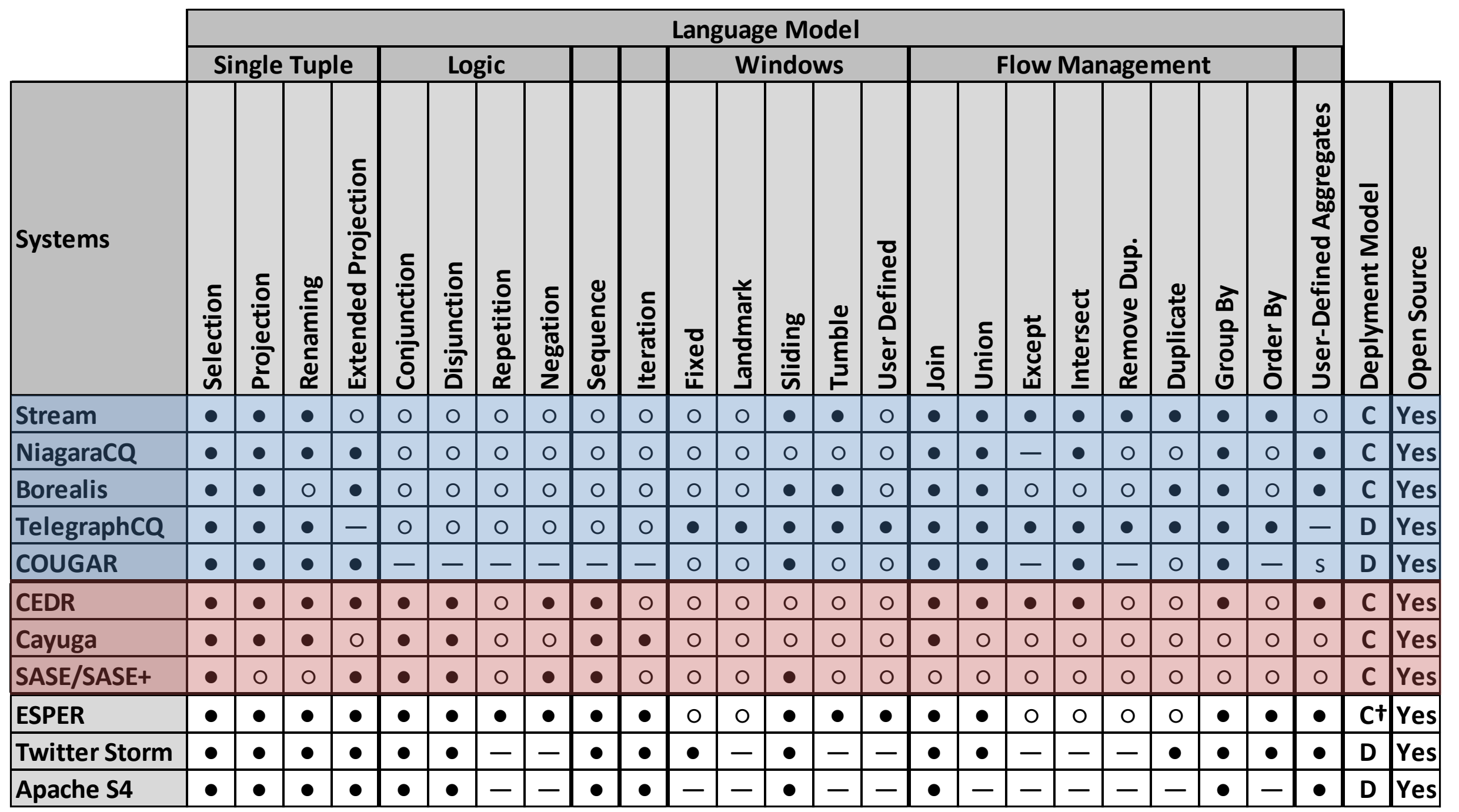




\section{Related Work}

Data Stream

Event Stream

Data + Event Stream

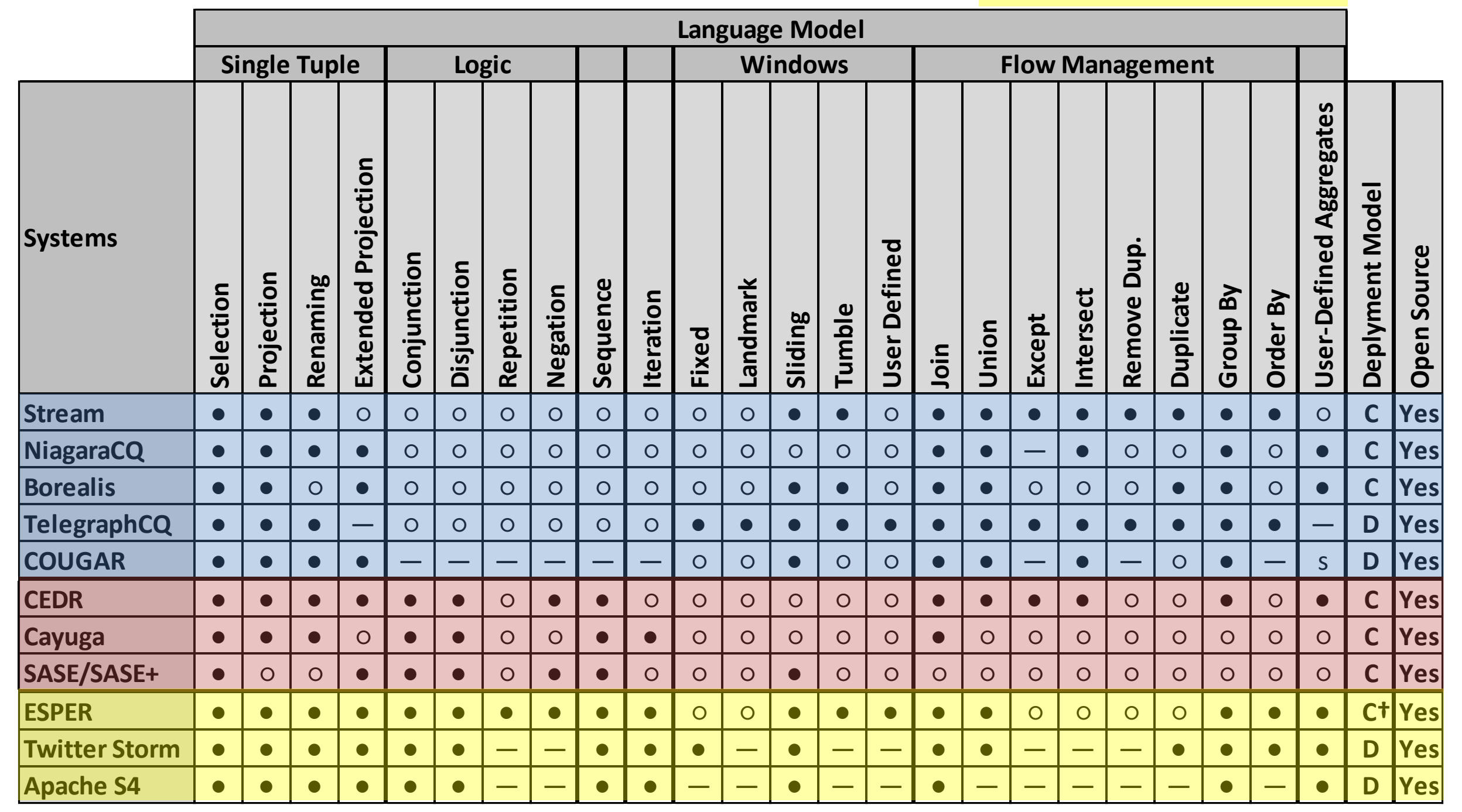




\section{Related Work}

\section{Data Stream}

\section{Event Stream}

Data + Event Stream

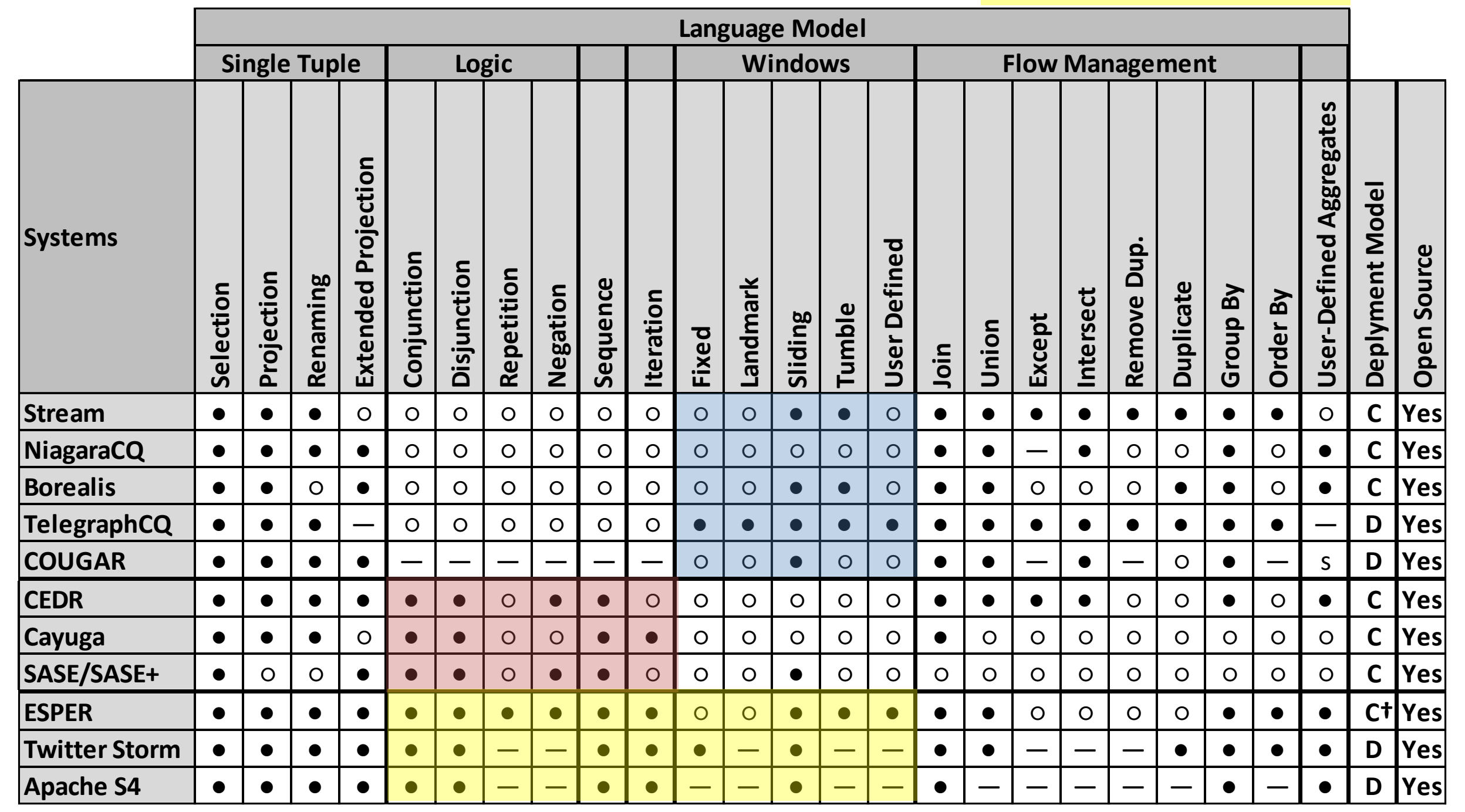




\section{Related Work}

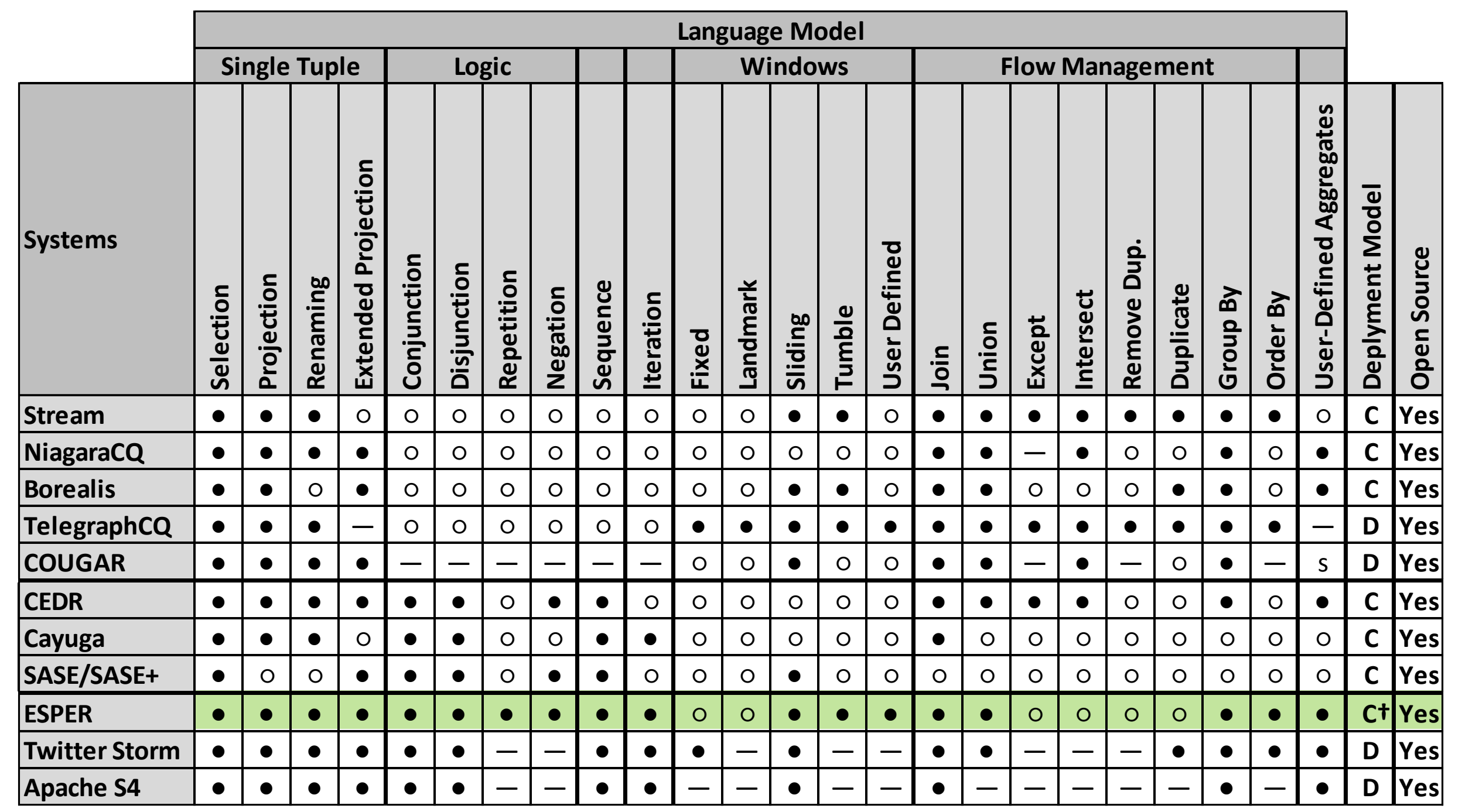




\section{Related Work}

- Data Management Systems (DSMSs)

- Properly designed to process huge amonts of data streams with the lowest possible latency

- Store queries (instead of data), receive data (instead of queries)

\section{DBMS Approach}

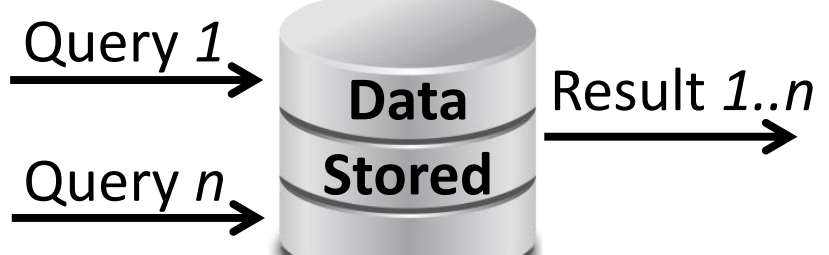

DSMS Approach

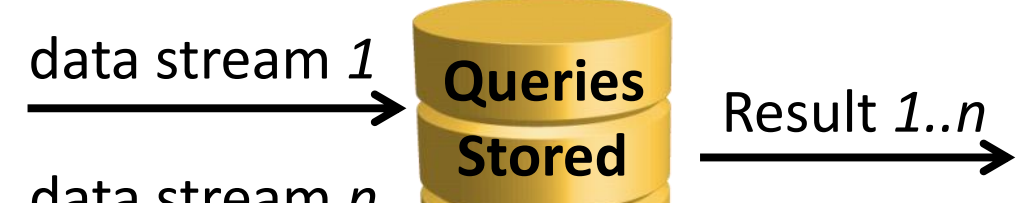

